\title{
Periodic Water Waves through A Heterogeneous Coastal Forest of Arbitrary Shape
}

\author{
Che-Wei Chang ${ }^{1, *}$, Philip L.-F. Liu ${ }^{1,2,3}$, Chiang C. Mei ${ }^{4}$, Maria Maza ${ }^{5}$ \\ ${ }^{1}$ School of Civil and Environmental Engineering, Cornell University, USA \\ ${ }^{2}$ Department of Civil and Environmental Engineering, National University of Singapore, Singapore \\ ${ }^{3}$ Institute of Oceanic and Hydrological Sciences, National Central University, Taiwan \\ ${ }^{4}$ Department of Civil and Environmental Engineering, MIT, USA \\ ${ }^{5}$ Instituto de Hidraulica Ambiental, Universidad de Cantabria, Spain \\ * Corresponding Author (Email: cc2338@cornell.edu)
}

\begin{abstract}
Small-amplitude water waves propagating through a heterogeneous coastal forest of arbitrary shape is studied. Following the theoretical approach introduced by Liu, Chang, Mei, Lomonaco, Martin, and Maza (2015), the forest is modeled by an array of rigid and vertical cylinders. Assuming that the wavelength is much larger than the cylinder diameter and the cylinder spacing, a multi-scale perturbation theory of homogenization (Mei and Vernescu 2010) is applied to separate the micro-scale flow problem within a unit cell, containing one or more cylinders, from the macro-scale wave dynamics. The complex coefficients in the derived macro-scale governing equations are computed from the solutions of micro-scale problem, in which the macro-scale pressure gradients are the driven force. The boundary integral equation method is employed to solve the macro-scale wave dynamic problem where the forest has an arbitrary shape and is composed of multiple forest patches. Each forest patch can be divided into subzones according to different forest properties, such as the porosity and cylinder diameter. Each subzone is considered as a homogeneous forest region with a constant bulk eddy viscosity, which is determined by invoking the balance of the time-averaged dissipation rate and the rate of work done by wave forces. A computing program has been developed based on the present approach. The numerical model is checked with existing theoretical works and laboratory experiments. The numerical solutions compare almost perfectly with the semi-analytical solutions for a single circular forest reported in Liu et al. (2015). The numerical model is then applied to cases where the forest region is made of multiple circular patches. Experimental data for these cases (Maza 2015; Maza, Lara, and Losada 2016) is used to validate the numerical results. The comparison between model predictions and the experimental data is in reasonable agreement. The effectiveness of these two special forest configurations on wave attenuation is also discussed.
\end{abstract}

\section{Introduction}

Coastal vegetation, as a natural barrier of shore regions against ocean waves, has long been studied via laboratory/field observation, mathematical modeling and numerical simulation. Comprehensive reviews on most of the key literatures, for both long waves and short waves, have been provided in Mei et al. (2011, 2014). Among the existing literatures, physical modelings were mostly conducted in wave flumes to reduce experimental difficulties and complexities. However, vegetation patches with finite areas are more commonly observed in fields, which have begun drawing researchers' attention in recent years. For example, Truong et al. (2015) used three circular patches in their laboratory experiments to model coastal wetlands. The cylinder patches were built on the tops of artificial marsh mounds, which led to breaking waves and swash currents occurring on the slope of mounds and passing through the patches. The same type of vegetated patches was further studied numerically by Yang et al. (2015). Rip currents were observed within the channels between patches. Effects from bathymetry, vegetation and the spacing between patches were investigated. Wave dissipations by vegetation were also observed. Similar laboratory experiments were conducted by

(C) 2016. This manuscript version is made available under the Elsevier user license http://www.elsevier.com/open-access/userlicense/1.0/ 
Irish et al. (2014), placing multiple small cylinder patches with high porosity along a steep slope to study the run-up of tsunami. In addition to multiple discontinuous patches, a single vegetated area with finite extent was used in several numerical studies to investigate wave dissipation by vegetation. Ma et al. (2013) developed a numerical model to study surface wave attenuation through a rectangular patch on a slope and the nearshore circulation in the surf zone. Similar vegetation patch was used in Zhang et al. (2016) to simulate wave propagation and wave-induced current. Anderson and Smith (2015) used different numerical models to study the effects of a finite rectangular cylinder patch on wave dissipation. In most of the studies, an array of cylinders were usually used to mimic trees and mangroves due to their rigidity. On the other hand, some researchers used real vegetation, such as common types of wetland grasses, in their wave-flume experiments, e.g. Ozeren et al. 2014. More recently, Lara et al. (2016) proposed a guideline for large-scale physical modeling on wave and current interactions with real salt marsh vegetation patch, where the analysis was reported in Maza et al. (2015). Furthermore, several latest studies on wave-vegetation interactions may be worthy of mentioning here. For example, Chakrabarti et al. (2016) conducted large eddy simulation for waves through emergent cylinders, which provides detailed interpretations of wave forces on cylinders and flow fields in vicinity of cylinders. Field observations were also conducted to study wave attenuation by mangroves and the possible effects on sedimentation (e.g. Horstman et al. 2014).

The progress of recent studies has provided a better understanding about the role of coastal vegetation on shore protection. However, the shapes of vegetated area in most studies were impractical. Also, a tool for estimating wave attenuation by multiple patches of arbitrary shape is still unavailable. Therefore, following Mei et al. (2014) and Liu et al. (2015) the theoretical development on the interactions between waves and coastal vegetation is extended to a general forest region in this paper. It has been shown in the previous studies that the model based on multi-scale perturbation techniques (i.e. homogenization method) has the ability to estimate the effectiveness of a forest area with a simple geometry (e.g. a circular forest) on the dissipation of the incoming wave energy. To further extend the capability of the multi-scale model, the general forest configuration, which is composed of multiple forest patches as sketched in Fig.1a, is considered in this paper. Each patch has an arbitrary shape and can have several subzones (Fig.1b) according to different forest properties (e.g. vegetation type/size, porosity and planting pattern). As shown in Fig.1c, a subzone can be surrounded by other subzones and/or the open water region. To resolve the arbitrary geometry of patches and the interfaces among subzones, the boundary integral equation method is employed to solve the macro-scale problem numerically.

Boundary integral equation methods have been widely applied to porous media flow problems (e.g. Liggett and Liu 1983), wave propagation and wave scattering problems, (e.g. Lee and Ayer 1981, Williams 1990 and Williams and Vazquez 1991). The boundary integral equation method was also used to study the wave oscillations and resonance behaviors in harbors with an arbitrary geometry (Lee 1971). One of the advantages of using this numerical method is that it reduces the dimension of the boundary value problem (BVP) by one. Solutions to the BVP are first provided along the boundaries of the flow domain. The solutions inside the domain can then be obtained by boundary integral formulas. The boundary integral equations for macro-scale wave solutions within the forest and the open water regions will be introduced in this paper. The matching conditions along the interface between adjacent subzones of a forest patch and that between the forest region and the open water will be presented as well.

In Liu et al. (2015) the eddy viscosity was assumed as a bulk value in the entire forest region. In this paper multiple subzones within each forest patch, according to the forest properties, are introduced, i.e. Fig.1. The energy balance concept and the drag coefficient formula suggested by Liu et al. (2015) are used to determine the bulk eddy viscosity within each subzone.

On the basis of the present approach, a numerical model is developed for computing the macro-scale wave solutions for both forest and open water region in conjunction of a micro-scale problem solver. To check the present model, we first re-examine the case with a single circular forest studied in Liu et al. (2015). Only negligible differences are observed between the present numerical results and the semi-analytical solutions. To further validate the present model, the laboratory experiments on multiple circular patches (Maza 2015; Maza et al. 2016) are investigated. Reasonable agreement between the experimental data and the model predictions is observed. The comparison of the effectiveness of a circular forest and multiple smaller patches in terms of wave damping and scattering is also presented. 
(a) Forest region

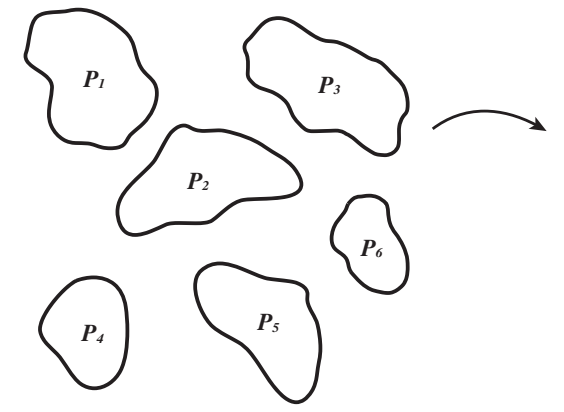

(b)

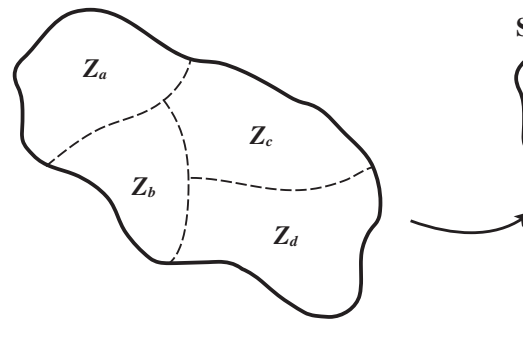

(c)

Forest subzone

Figure 1: Sketch of a typical coastal forest configuration: (a) a forest region - consists of multiple patches with arbitrary shape; (b) one of the forest patches (e.g. Patch 3) - can be composed of several subzones; (c) one of the subzones (e.g. Subzone a) within Patch $3-\mathrm{S}$ denotes the boundary between the subzone and open water, while D shows the interface with adjacent subzone.

\section{Formulation}

The theoretical development and formulation of the homogenization theory being applied to the wave-coastal forest interaction problem have been given in Liu et al. (2015). However, for clarity some of the key concepts and formulas are summarized herein. We consider small-amplitude periodic waves incident upon a coastal forest with arbitrary shape. In a constant water depth $h_{0}$ the coastal forest is modeled by rigid and vertical cylinders with characteristic spacing $\ell$. For the incident waves of the characteristic frequency $\omega$ and amplitude $A$, the typical wavelength is $1 / k$ with $k$ being the characteristic wavenumber. The dimensionless physical variables can be defined as follows,

$$
x_{i}^{*}=\frac{x_{i}}{\ell}, \quad z^{*}=k z, \quad h^{*}=k h, \quad \eta^{*}=\frac{\eta}{A}, \quad t^{*}=t \omega,
$$

and

$$
p^{*}=\frac{p}{\rho g A}, \quad u_{i}^{*}=\frac{u_{i}}{A \omega}, \quad w^{*}=\frac{w}{A \omega}
$$

where the horizontal coordinates $\vec{x}=\left(x_{1}, x_{2}\right)$ are normalized by the cylinder spacing $\ell$ while the vertical coordinate $z$ by the wavelength $1 / k$. The free surface displacement is represented by $\eta$ and scaled by the wave amplitude $A$. Considering the wavelength in the compatible order of magnitude as the water depth, i.e. $k h=\mathcal{O}(1)$, but greater than the cylinder spacing, we define a small parameter as

$$
\varepsilon=k \ell \ll \mathcal{O}(1) \text {. }
$$

Two horizontal coordinates, $x_{i}^{*}$ and $X_{i}^{*}$, are introduced, separating these two representative length scales:

$$
X_{i}^{*}=\varepsilon x_{i}^{*}=k x_{i}, \quad Z^{*} \equiv z^{*}=k z .
$$

From here on, only dimensionless variables will be used and the asterisks $(\cdot)^{*}$ will be omitted for brevity. Using the multiple-scale perturbation method with the small parameter in (2.3), all the dimensionless physical variables can be expanded as

$$
\eta=\eta^{(0)}+\varepsilon \eta^{(1)}+\varepsilon^{2} \eta^{(2)}+\cdots, \quad p=p^{(0)}+\varepsilon p^{(1)}+\varepsilon^{2} p^{(2)}+\cdots,
$$

and

$$
u_{i}=u_{i}^{(0)}+\varepsilon u_{i}^{(1)}+\varepsilon^{2} u_{i}^{(2)}+\cdots, \quad w=w^{(0)}+\varepsilon w^{(1)}+\varepsilon^{2} w^{(2)}+\cdots,
$$

where the variables of all the orders are functions of $\left(x_{i}, X_{i}, Z, t\right)$. For periodic waves, we can express the variables as

$$
F\left(x_{i}, X_{i}, Z, t\right)=\Re\left\{\tilde{F}\left(x_{i}, X_{i}, Z\right) e^{-\mathrm{i} t}\right\}
$$

where $\tilde{F}$ is the spatially dependent amplitude function and the operator $\Re(\cdot)$ denotes that only the real part is considered. 


\subsection{Micro-scale (cell) Problem}

Focusing on the flow motion in a micro-scale cell with one or more cylinders inside, the leading-order velocity and the pressure fluctuation can both be expressed in terms of the leading-order pressure gradient (Mei et al. 2011, 2014) as follows,

$$
\tilde{u}_{i}^{(0)}=-\tilde{K}_{i j}(\vec{x}) \frac{\partial \tilde{p}^{(0)}}{\partial X_{j}}, \quad \tilde{w}^{(0)}=-\tilde{W}(\vec{x}) \frac{\partial \tilde{p}^{(0)}}{\partial Z}, \quad \tilde{p}^{(1)}=-\tilde{A}_{j}(\vec{x}) \frac{\partial \tilde{p}^{(0)}}{\partial X_{j}}, \quad \vec{x} \in \Omega_{\mathrm{f}}
$$

where $\Omega_{\mathrm{f}}$ represents the fluid part within a unit cell $\Omega$. The governing equations for the cell problem can then be derived from the mass conservation of $\mathcal{O}(1)$ and the momentum equations of $\mathcal{O}(\epsilon)$ as presented in Liu et al. (2015):

$$
\frac{\partial \tilde{K}_{i j}}{\partial x_{i}}=0, \quad-\mathrm{i} \tilde{K}_{i j}=\delta_{i j}-\frac{\partial \tilde{A}_{j}}{\partial x_{i}}+\frac{\partial \tilde{\tau}_{i k}^{(j)}}{\partial x_{k}}, \quad \vec{x} \in \Omega_{\mathrm{f}}
$$

and

$$
-\mathrm{i} \tilde{W}=1+\sigma \frac{\partial^{2} \tilde{W}}{\partial x_{j} \partial x_{j}}, \quad \vec{x} \in \Omega_{\mathrm{f}}
$$

in which the stress tensor is

$$
\tilde{\tau}_{i k}^{(j)}=\sigma\left(\frac{\partial \tilde{K}_{i j}}{\partial x_{k}}+\frac{\partial \tilde{K}_{k j}}{\partial x_{i}}\right) \quad \text { with } \quad \sigma=\frac{\nu_{e}}{\omega \ell^{2}}
$$

where $\nu_{e}$ denotes the dimensional eddy viscosity. Note that $\tilde{K}_{i j}$ and $\tilde{A}_{j}$ vary only horizontally. In this paper we propose that each subzone of the forest region is considered as a homogeneous forest area where a bulk eddy viscosity can be assumed. Following Liu et al. (2015), the dimensionless bulk eddy viscosity $(\sigma)$ within each subzone (e.g. subzone $\xi$ ) is determined by the concept of energy balance with area integral over the subzone instead of the entire forest region. Therefore, the values of eddy viscosity can be different for different subzones and the corresponding cell problem has to be solved independently. Other details of the numerical solutions to the cell problem can be found in Liu et al. (2015) and Mei et al. (2014).

\subsection{Macro-scale (wavelength-scale) Problem}

In Liu et al. (2015), the leading-order cell-averaged equation governing the macro-scale wave dynamics has been derived and expressed in terms of a complex potential $\phi=-\mathrm{i} \tilde{p}^{(0)}$ as

$$
\frac{\partial}{\partial X_{i}}\left[n \frac{\partial \phi}{\partial X_{i}}+M_{i k} \frac{\partial \phi}{\partial X_{k}}\right]+(n+N) \frac{\partial^{2} \phi}{\partial Z^{2}}=0, \quad i \& k=1,2
$$

where the cell porosity is defined as $n=\Omega_{\mathrm{f}} / \Omega$. The complex coefficients $M_{i k}$ and $N$ are both determined by the cell problem solutions:

$$
M_{i k}=\frac{1}{\Omega} \oint_{S_{c}}\left[-\tilde{A}_{k} \delta_{i j}+\sigma\left(\frac{\partial \tilde{K}_{i k}}{\partial x_{j}}+\frac{\partial \tilde{K}_{j k}}{\partial x_{i}}\right)\right] n_{j}^{S_{c}} d s, \quad N=\frac{1}{\Omega} \sigma \oint_{S_{c}}\left(\frac{\partial \tilde{W}}{\partial x_{j}} n_{j}^{S_{c}}\right) d s
$$

in which $n_{j}^{S_{c}}$ denotes the unit normal vector to the cylinder surface $S_{c}$. The eddy viscosity $\sigma$ is assumed as a constant bulk value within a subzone of a forest patch such that the complex coefficients in (2.13) remain constant. Thus, for each subzone (2.12) becomes

$$
\frac{\partial^{2} \phi}{\partial X_{i} \partial X_{i}}+\left(\frac{n+N}{n+M}\right) \frac{\partial^{2} \phi}{\partial Z^{2}}=0, \quad i=1,2
$$

in which $M_{11}=M_{22} \equiv M$ and $M_{12}=M_{21}=0$ are applied. Outside of the forest, the complex coefficients would vanish (i.e., $M=N=0$ ) and the porosity reduces to unity (i.e. $n=1$ ). The detailed derivations and the corresponding boundary conditions can be found in Liu et al. (2015). 


\section{Boundary integral equation method}

Boundary integral equation method is employed to deal with the arbitrary shape and to solve the macro-scale problem numerically. In the following, the integral formulations for a subzone (e.g. Fig.1c) of a forest patch and for the open water region are introduced, respectively. The matching conditions along the interfaces of adjacent subzones and the boundary between the forest region and open water are presented as well.

\subsection{Integral equation formulation for a forest subzone}

First we consider a forest subzone $\xi$ with an arbitrary shape, e.g. Fig.1c. We note that the subzone could be surrounded by other subzones and/or the open water region. As shown in (2.14), the governing equation for the complex potential $\phi_{\xi}$ has

$$
\frac{\partial^{2} \phi_{\xi}}{\partial X_{1} \partial X_{1}}+\frac{\partial^{2} \phi_{\xi}}{\partial X_{2} \partial X_{2}}+\left[\frac{n_{\xi}+N_{\xi}}{n_{\xi}+M_{\xi}}\right] \frac{\partial^{2} \phi_{\xi}}{\partial Z^{2}}=0
$$

in which the complex coefficients $M_{\xi}$ and $N_{\xi}$ are obtained separately for each subzone $\xi$. Due to the arbitrary shape of forest subzone $\xi$, the solution to (3.1) can be expressed as

$$
\phi_{\xi}=\sum_{q=0}^{\infty} \mathcal{A}_{\xi_{q}}\left(\vec{X}_{i}\right) \frac{\cosh \hat{k}_{\xi_{q}}(Z+h)}{\cosh \hat{k}_{\xi_{q}} h}
$$

which separates the horizontal variables from the vertical variable with the corresponding wavenumber $\hat{k}_{\xi_{q}}$ being the complex roots of the dispersion relationship:

$$
1=\left[\frac{n_{\xi}+N_{\xi}}{n_{\xi}}\right] \hat{k}_{\xi_{q}} \tanh \hat{k}_{\xi_{q}} h .
$$

The horizontal function $\mathcal{A}_{\xi_{q}}$ in (3.2) satisfies the two-dimensional Helmholtz equation:

$$
\left(\frac{\partial^{2} \mathcal{A}_{\xi_{q}}}{\partial X_{1} \partial X_{1}}+\frac{\partial^{2} \mathcal{A}_{\xi_{q}}}{\partial X_{2} \partial X_{2}}\right)+\left(\gamma_{\xi} \hat{k}_{\xi_{q}}\right)^{2} \mathcal{A}_{\xi_{q}}=0 \quad \text { with } \quad \gamma_{\xi}=\sqrt{\left(n_{\xi}+N_{\xi}\right) /\left(n_{\xi}+M_{\xi}\right)},
$$

which can be converted into the following integral equation (Liggett and Liu 1983):

$$
\mathcal{A}_{\xi_{q}}\left(\vec{X}_{i}\right)=\frac{-\mathrm{i} \pi}{2 \alpha} \int_{\mathrm{S}_{\xi}+\mathrm{D}_{\xi \psi}}\left\{\mathcal{A}_{\xi_{q}}\left(\vec{X}_{j}\right) \frac{\partial\left[H_{0}^{(1)}\left(\gamma_{\xi} \hat{k}_{\xi_{q}} \underline{r}\right)\right]}{\partial \hat{n}_{\xi}}-H_{0}^{(1)}\left(\gamma_{\xi} \hat{k}_{\xi_{q}} \underline{r}\right) \frac{\partial \mathcal{A}_{\xi_{q}}\left(\vec{X}_{j}\right)}{\partial \hat{n}_{\xi}}\right\} d S
$$

where the boundary of subzone $\xi$ can include the boundary $\mathrm{S}_{\xi}$ to the open water and/or the interfaces $\mathrm{D}_{\xi \psi}$ with adjacent zones $\psi$. In the integral equation, $\vec{X}_{i}$ denotes the coordinate of a point located on the boundary of the subzone (i.e. $\vec{X}_{i} \in \mathrm{S}_{\xi} / \mathrm{D}_{\xi \psi}$ ) where its interior angle $\alpha$ (Fig.2) has to be taken into account. $\vec{X}_{j}$ denotes another boundary point of the subzone with $\underline{r}=\left|\vec{X}_{i}-\vec{X}_{j}\right|$ giving the distance between two boundary points. $H_{0}^{(1)}$ is the Hankel function of the first kind and $\hat{n}_{\xi}$ represents the outward normal direction to the boundary of subzone $\xi$. Therefore, the integral equation (3.5) can be used to solve unknown $\mathcal{A}_{\xi_{q}}$ and its normal derivative along the boundary. Once the solutions along the forest boundary are obtained, the wave solutions inside the forest can be found by assigning $\vec{X}_{i}$ as an interior point and the interior angle $=2 \pi$ :

$$
\mathcal{A}_{\xi_{q}}\left(\vec{X}_{i}\right)=\frac{-\mathrm{i}}{4} \int_{\mathrm{S}_{\xi}+\mathrm{D}_{\xi \psi}}\left\{\mathcal{A}_{\xi_{q}}\left(\vec{X}_{j}\right) \frac{\partial\left[H_{0}^{(1)}\left(\gamma_{\xi} \hat{k}_{\xi_{q}}\right)\right]}{\partial \hat{n}_{\xi}}-H_{0}^{(1)}\left(\gamma_{\xi} \hat{k}_{\xi_{q}} \underline{r}\right) \frac{\partial \mathcal{A}_{\xi_{q}}\left(\vec{X}_{j}\right)}{\partial \hat{n}_{\xi}}\right\} d S
$$

in which the point $\vec{X}_{i}$ is an interior point in subzone $\xi$ while $\vec{X}_{j}$ are on the boundary of subzone $\xi$. 


\subsection{Integral equation formulation for open water}

In the open water region (with subscript $\mathrm{S}$ ), the equation governing for the complex potential $\phi_{\mathrm{S}}$ is

$$
\frac{\partial^{2} \phi_{\mathrm{S}}}{\partial X_{1} \partial X_{1}}+\frac{\partial^{2} \phi_{\mathrm{S}}}{\partial X_{2} \partial X_{2}}+\frac{\partial^{2} \phi_{\mathrm{S}}}{\partial Z^{2}}=0
$$

and its solution, including both incident and scattered waves, can be expressed as

$$
\phi_{\mathrm{S}}=A_{0} \frac{\cosh k_{0}(Z+h)}{\cosh k_{0} h} e^{\mathrm{i} k_{0} X_{1 i}}+\sum_{p=0}^{\infty} \mathcal{B}_{p}\left(\vec{X}_{i}\right) \frac{\cosh k_{p}(Z+h)}{\cosh k_{p} h}
$$

where $A_{0}=-\mathrm{i}$ and $k_{p}$ satisfies the dispersion relationship:

$$
1=k_{p} \tanh k_{p} h
$$

with one real root $k_{0}$ and infinite number of imaginary roots $k_{p}$ (when $p \geq 1$ ). Assuming the incident waves in $X_{1}$ direction, $X_{1 i}$ in (3.8) denotes the $X_{1}$ coordinate of point $i$. The corresponding Helmholtz equation for $\mathcal{B}_{p}$ is

$$
\left(\frac{\partial^{2} \mathcal{B}_{p}}{\partial X_{1} \partial X_{1}}+\frac{\partial^{2} \mathcal{B}_{p}}{\partial X_{2} \partial X_{2}}\right)+k_{p}^{2} \mathcal{B}_{p}=0
$$

which can also be converted into the following integral equation:

$$
\mathcal{B}_{p}\left(\vec{X}_{i}\right)=\frac{-\mathrm{i} \pi}{2(2 \pi-\alpha)} \int_{\mathrm{S}}\left\{\mathcal{B}_{p}\left(\vec{X}_{j}\right) \frac{\partial\left[H_{0}^{(1)}\left(k_{p} \underline{r}\right)\right]}{\partial \hat{n}_{\mathrm{S}}}-H_{0}^{(1)}\left(k_{p} \underline{r}\right) \frac{\partial \mathcal{B}_{p}\left(\vec{X}_{j}\right)}{\partial \hat{n}_{\mathrm{S}}}\right\} d S
$$

where $\vec{X}_{i}$ and $\vec{X}_{j}$ are both on the boundary between the open water and all the forest patches and $\mathrm{S}$ in (3.11) represents the combination of the boundaries of all the forest patches. Likewise, once $\mathcal{B}_{p}$ along the boundary of all the forest patches is found, the wave solutions can be obtained from (3.8) with (3.11) being modified as

$$
\mathcal{B}_{p}\left(\vec{X}_{i}\right)=\frac{-\mathrm{i}}{4} \int_{\mathrm{S}}\left\{\mathcal{B}_{p}\left(\vec{X}_{j}\right) \frac{\partial\left[H_{0}^{(1)}\left(k_{p} \underline{r}\right)\right]}{\partial \hat{n}_{\mathrm{S}}}-H_{0}^{(1)}\left(k_{p} \underline{r}\right) \frac{\partial \mathcal{B}_{p}\left(\vec{X}_{j}\right)}{\partial \hat{n}_{\mathrm{S}}}\right\} d S
$$

in which $\vec{X}_{i}$ is assigned at any location within the open water region (i.e. an interior point of an unbounded area) while $\vec{X}_{j}$ are on the boundary between the open water and the forest region. Note again that the outward normal directions of the boundary with respect to the forest region and the open water are opposite.

\subsection{Matching conditions along the boundaries}

The wave solutions along the boundaries between two subzones, and those between subzones and the open water region can be found from (3.5) and (3.11) if matching conditions along the boundaries are prescribed. As noted, a forest subzone can have its boundary in contact with open water and/or adjacent subzones. Hereafter the former will be referred as the exterior boundary of a forest subzone while the latter as the interface with other zones. In both situations the continuity of pressure and normal mass fluxes are imposed.

\subsubsection{Boundary between forest and open water}

We consider first the exterior boundary. The pressure continuity provides

$$
\phi_{\xi}=\phi_{\mathrm{S}}, \quad-h<Z<0
$$

i.e.

$$
\sum_{q=0}^{\infty} \mathcal{A}_{\xi_{q}} \frac{\cosh \hat{k}_{\xi_{q}}(Z+h)}{\cosh \hat{k}_{\xi_{q}} h}=A_{0} \frac{\cosh k_{0}(Z+h)}{\cosh k_{0} h} e^{\mathrm{i} k_{0} X_{1 i}}+\sum_{p=0}^{\infty} \mathcal{B}_{p} \frac{\cosh k_{p}(Z+h)}{\cosh k_{p} h}
$$


The normal fluxes must be continuous along the forest boundary as well:

$$
\left(n_{\xi}+M_{\xi}\right) \frac{\partial \phi_{\xi}}{\partial \hat{n}_{\xi}}=\frac{\partial \phi_{\mathrm{S}}}{\partial \hat{n}_{\xi}}, \quad-h<Z<0
$$

i.e.

$$
\left(n_{\xi}+M_{\xi}\right) \sum_{q=0}^{\infty} \frac{\partial \mathcal{A}_{\xi_{q}}}{\partial \hat{n}_{\xi}} \frac{\cosh \hat{k}_{\xi_{q}}(Z+h)}{\cosh \hat{k}_{\xi_{q}} h}=A_{0} \frac{\cosh k_{0}(Z+h)}{\cosh k_{0} h} \frac{\partial e^{\mathrm{i} k_{0} X_{1 i}}}{\partial \hat{n}_{\xi}}+\sum_{p=0}^{\infty} \frac{\partial \mathcal{B}_{p}}{\partial \hat{n}_{\xi}} \frac{\cosh k_{p}(Z+h)}{\cosh k_{p} h} .
$$

where (3.2) and (3.8) have been used. Applying the orthogonality of hyperbolic cosine function, where the definition can be found in Liu et al. (2015)'s (A.3), to the above (3.14) and (3.16) yields

$$
\mathcal{A}_{\xi_{q}} \frac{\Pi\left(\hat{k}_{\xi_{q}}\right)}{\cosh \hat{k}_{\xi_{q}} h}=A_{0} \frac{\Gamma_{0 \xi_{q}}}{\cosh k_{0} h} e^{\mathrm{i} k_{0} X_{1 i}}+\sum_{p=0}^{\infty} \mathcal{B}_{p} \frac{\Gamma_{p \xi_{q}}}{\cosh k_{p} h}
$$

and

$$
\left(n_{\xi}+M_{\xi}\right) \sum_{q=0}^{\infty} \frac{\partial \mathcal{A}_{\xi_{q}}}{\partial \hat{n}_{\xi}} \frac{\Gamma_{\xi_{q} p}}{\cosh \hat{k}_{\xi_{q}} h}=A_{0} \delta_{0 p} \frac{\Pi\left(k_{0}\right)}{\cosh k_{0} h} \frac{\partial e^{\mathrm{i} k_{0} X_{1 i}}}{\partial \hat{n}_{\xi}}+\frac{\partial \mathcal{B}_{p}}{\partial \hat{n}_{\xi}} \frac{\Pi\left(k_{p}\right)}{\cosh k_{p} h}
$$

in which $\delta_{0 p}$ denotes the Kronecker delta with nonzero value only when $p=0$ (i.e. $\delta_{0 p}=1$ when $p=0$ ), while $\Pi\left(\hat{k}_{\xi_{q}}\right)$ and $\Gamma_{p \xi_{q}}$ are defined as

$$
\Pi\left(\hat{k}_{\xi_{q}}\right)=\frac{h}{2}\left(1+\frac{\sinh 2 \hat{k}_{\xi_{q}} h}{2 \hat{k}_{\xi_{q}} h}\right), \quad \Gamma_{p \xi_{q}}=\frac{k_{p} \sinh \left(k_{p} h\right) \cosh \left(\hat{k}_{\xi_{q}} h\right)-\hat{k}_{\xi_{q}} \cosh \left(k_{p} h\right) \sinh \left(\hat{k}_{\xi_{q}} h\right)}{k_{p}^{2}-\hat{k}_{\xi_{q}}^{2}} .
$$

\subsubsection{Interface between forest subzones}

Likewise, along the interface between two subzones (i.e. $\mathrm{D}_{\xi \psi}$ with $\xi$ and $\psi$ denoting two adjacent zones), the pressure continuity yields

$$
\phi_{\xi}=\phi_{\psi}, \quad-h<Z<0
$$

Substituting (3.2) into the above condition gives

$$
\sum_{q=0}^{\infty} \mathcal{A}_{\xi_{q}}\left(\vec{X}_{i}\right) \frac{\cosh \hat{k}_{\xi_{q}}(Z+h)}{\cosh \hat{k}_{\xi_{q}} h}=\sum_{r=0}^{\infty} \mathcal{A}_{\psi_{r}}\left(\vec{X}_{i}\right) \frac{\cosh \hat{k}_{\psi_{r}}(Z+h)}{\cosh \hat{k}_{\psi_{r}} h} .
$$

Also, the continuity of normal fluxes is required:

$$
\left(n_{\xi}+M_{\xi}\right) \frac{\partial \phi_{\xi}}{\partial \hat{n}_{\xi}}=-\left(n_{\psi}+M_{\psi}\right) \frac{\partial \phi_{\psi}}{\partial \hat{n}_{\psi}}, \quad-h<Z<0
$$

i.e.

$$
\left(n_{\xi}+M_{\xi}\right) \sum_{q=0}^{\infty} \frac{\partial \mathcal{A}_{\xi_{q}}}{\partial \hat{n}_{\xi}} \frac{\cosh \hat{k}_{\xi_{q}}(Z+h)}{\cosh \hat{k}_{\xi_{q}} h}=-\left(n_{\psi}+M_{\psi}\right) \sum_{r=0}^{\infty} \frac{\partial \mathcal{A}_{\psi_{r}}}{\partial \hat{n}_{\psi}} \frac{\cosh \hat{k}_{\psi_{r}}(Z+h)}{\cosh \hat{k}_{\psi_{r}} h} .
$$

Again, applying the orthogonality of $\cosh (\cdot)$ to (3.21) and (3.23) yields

$$
\mathcal{A}_{\xi_{q}} \frac{\Pi\left(\hat{k}_{\xi_{q}}\right)}{\cosh \hat{k}_{\xi_{q}} h}=\sum_{r=0}^{\infty} \mathcal{A}_{\psi_{r}} \frac{\Gamma_{\psi_{r} \xi_{q}}}{\cosh \hat{k}_{\psi_{r}} h}
$$

and

$$
\left(n_{\xi}+M_{\xi}\right) \sum_{q=0}^{\infty} \frac{\partial \mathcal{A}_{\xi_{q}}}{\partial \hat{n}_{\xi}} \frac{\Gamma_{\xi_{q} \psi_{r}}}{\cosh \hat{k}_{\xi_{q}} h}=-\left(n_{\psi}+M_{\psi}\right) \frac{\partial \mathcal{A}_{\psi_{r}}}{\partial \hat{n}_{\psi}} \frac{\Pi\left(\hat{k}_{\psi_{r}}\right)}{\cosh \hat{k}_{\psi_{r}} h} .
$$

Equations (3.17), (3.18) along with (3.24), (3.25) are to be used for solving unknown $\mathcal{A}_{\xi_{q}}, \mathcal{A}_{\psi_{r}}$ and $\mathcal{B}_{p}$ as well as their normal derivatives on the exterior boundary of the forest and the interface between subzones. 


\subsection{Numerical implementation}

In the numerical computations, the boundary of a forest subzone $\xi$ is discretized into elements. Assuming uniform-distributed solution along each element of length $\Delta S$ with $\vec{X}_{j}$ denoting its middle point, we express (3.5) in the discrete form as:

$$
\mathcal{A}_{\xi_{q}}\left(\vec{X}_{i}\right)=\frac{-\mathrm{i} \pi}{2 \alpha} \sum_{j=1}^{\mathbb{N}_{\xi}}\left\{\mathcal{A}_{\xi_{q}}\left(\vec{X}_{j}\right) \frac{\partial\left[H_{0}^{(1)}\left(\gamma_{\xi} \hat{k}_{\xi_{q}}\right)\right]}{\partial \hat{n}_{\xi}}-H_{0}^{(1)}\left(\gamma_{\xi} \hat{k}_{\xi_{q}} \underline{r}\right) \frac{\partial \mathcal{A}_{\xi_{q}}\left(\vec{X}_{j}\right)}{\partial \hat{n}_{\xi}}\right\} \Delta S
$$

in which $\mathbb{N}_{\xi}$ denotes the total number of elements along the boundary of subzone $\xi$, which can include its exterior boundary $\left(\mathrm{S}_{\xi}\right)$ to open water and/or interfaces $\left(\mathrm{D}_{\xi \psi}\right)$ with other subzones. Equation (3.26) can be written by index notation as:

$$
\left(\mathcal{A}_{\xi_{q}}\right)_{i}+\left(\overline{\mathcal{C}}_{\xi_{q}}\right)_{i j}\left(\mathcal{A}_{\xi_{q}}\right)_{j}=\left(\overline{\mathcal{D}}_{\xi_{q}}\right)_{i j}\left(\frac{\partial \mathcal{A}_{\xi_{q}}}{\partial \hat{n}_{\xi}}\right)_{j} \quad \text { with } \quad i, j=1 \cdots \mathbb{N}_{\xi}
$$

where

$$
\left(\overline{\mathcal{C}}_{\xi_{q}}\right)_{i j}=\frac{\mathrm{i} \pi}{2 \alpha}\left[\left(-\gamma_{\xi} \hat{k}_{\xi_{q}}\right) H_{1}^{(1)}\left(\gamma_{\xi} \hat{k}_{\xi_{q}} \underline{r}_{i j}\right)\right] \frac{\partial \underline{r}_{i j}}{\partial \hat{n}_{\xi}} \Delta S, \quad\left(\overline{\mathcal{D}}_{\xi_{q}}\right)_{i j}=\frac{\mathrm{i} \pi}{2 \alpha} H_{0}^{(1)}\left(\gamma_{\xi} \hat{k}_{\xi_{q}} \underline{r}_{i j}\right) \Delta S .
$$

We can also rearrange (3.27) and express the unknown $\mathcal{A}_{\xi_{q}}$ at the boundary point $\vec{X}_{i}$ in terms of the normal derivatives of $\mathcal{A}_{\xi_{q}}$ along the entire boundary of the subzone:

$$
\left(\mathcal{A}_{\xi_{q}}\right)_{i}=\left(\overline{\mathcal{G}}_{\xi_{q}}\right)_{i j}\left(\frac{\partial \mathcal{A}_{\xi_{q}}}{\partial \hat{n}_{\xi}}\right)_{j} \quad \text { with } \quad i, j=1 \cdots \mathbb{N}_{\xi}
$$

in which the coefficient matrix is obtained by $\left[\overline{\mathcal{G}}_{\xi_{q}}\right]=\left[\mathcal{I}+\overline{\mathcal{C}}_{\xi_{q}}\right]^{-1}\left[\overline{\mathcal{D}}_{\xi_{q}}\right]$ with $\mathcal{I}$ being the identity matrix.

For open water, on the other hand, (3.11) in the discrete form is as follows,

$$
\mathcal{B}_{p}\left(\vec{X}_{i}\right)=\frac{-\mathrm{i} \pi}{2(2 \pi-\alpha)} \sum_{j=1}^{\mathbb{N}_{\mathrm{S}}}\left\{\mathcal{B}_{p}\left(\vec{X}_{j}\right) \frac{\partial\left[H_{0}^{(1)}\left(k_{p} \underline{r}\right)\right]}{\partial \hat{n}_{\mathrm{S}}}-H_{0}^{(1)}\left(k_{p} \underline{\underline{r}}\right) \frac{\partial \mathcal{B}_{p}\left(\vec{X}_{j}\right)}{\partial \hat{n}_{\mathrm{S}}}\right\} \Delta S
$$

where $\mathbb{N}_{S}$ gives the total number of elements along the boundary that separates all the forest patches from the open water region, i.e. the exterior boundaries of all the forest subzones (i.e. $\mathrm{S}=\sum \mathrm{S}_{\xi}$ ). Again, we rearrange the above equation and express it by index notation:

$$
\left(\mathcal{B}_{p}\right)_{i}+\left(\overline{\mathcal{E}}_{p}\right)_{i j}\left(\mathcal{B}_{p}\right)_{j}=\left(\overline{\mathcal{F}}_{p}\right)_{i j}\left(\frac{\partial \mathcal{B}_{p}}{\partial \hat{n}_{\mathrm{S}}}\right)_{j} \quad \text { with } \quad i, j=1 \cdots \mathbb{N}_{\mathrm{S}}
$$

where

$$
\left(\overline{\mathcal{E}}_{p}\right)_{i j}=\frac{\mathrm{i} \pi}{2(2 \pi-\alpha)}\left[-k_{p} H_{1}^{(1)}\left(k_{p} \underline{r}_{i j}\right)\right] \frac{\partial \underline{r}_{i j}}{\partial \hat{n}_{\mathrm{S}}} \Delta S, \quad\left(\overline{\mathcal{F}}_{p}\right)_{i j}=\frac{\mathrm{i} \pi}{2(2 \pi-\alpha)} H_{0}^{(1)}\left(k_{p} \underline{r}_{i j}\right) \Delta S .
$$

We can also express the unknown function $\mathcal{B}_{p}$ in terms of its normal derivative along the boundaries:

$$
\left(\mathcal{B}_{p}\right)_{i}=\left(\overline{\mathcal{Q}}_{p}\right)_{i j}\left(\frac{\partial \mathcal{B}_{p}}{\partial \hat{n}_{\mathrm{S}}}\right)_{j} \text { with } \quad i, j=1 \cdots \mathbb{N}_{\mathrm{S}}
$$

where $\left[\overline{\mathcal{Q}}_{p}\right]=\left[\mathcal{I}+\overline{\mathcal{E}}_{p}\right]^{-1}\left[\overline{\mathcal{F}}_{p}\right]$ denotes the coefficient matrix.

To solve the unknown functions $\mathcal{A}_{\xi_{q}}$ and $\mathcal{B}_{p}$ and their normal derivatives, the matching conditions introduced in Sec.3.3 must be applied. As specified in (3.17) and (3.18), along the exterior boundary of a forest subzone in contact with open water (i.e. $\vec{X}_{i} \in \mathrm{S}_{\xi}$ ), the continuity of pressure and normal fluxes yields

$$
\frac{\Pi\left(\hat{k}_{\xi_{q}}\right)}{\cosh \hat{k}_{\xi_{q}} h}\left(\overline{\mathcal{G}}_{\xi_{q}}\right)_{i j}\left(\frac{\partial \mathcal{A}_{\xi_{q}}}{\partial \hat{n}_{\xi}}\right)_{j}=A_{0} \frac{\Gamma_{0 \xi_{q}}}{\cosh k_{0} h}\left(e^{\mathrm{i} k_{0} X_{1}}\right)_{i}+\sum_{p=0}^{\infty} \frac{\Gamma_{p \xi_{q}}}{\cosh k_{p} h}\left(\overline{\mathcal{Q}}_{p}\right)_{i m}\left(\frac{\partial \mathcal{B}_{p}}{\partial \hat{n}_{\mathrm{S}}}\right)_{m}
$$


in which the substitution of (3.29) and (3.33) has been made, and

$$
\left(n_{\xi}+M_{\xi}\right) \sum_{q=0}^{\infty} \frac{\Gamma_{\xi_{q} p}}{\cosh \hat{k}_{\xi_{q}} h}\left(\frac{\partial \mathcal{A}_{\xi_{q}}}{\partial \hat{n}_{\xi}}\right)_{i}=A_{0} \delta_{0 p} \frac{\Pi\left(k_{0}\right)}{\cosh k_{0} h}\left(\frac{\partial e^{i k_{0} X_{1}}}{\partial \hat{n}_{\xi}}\right)_{i}+\frac{\Pi\left(k_{p}\right)}{\cosh k_{p} h}\left(\frac{\partial \mathcal{B}_{p}}{\partial \hat{n}_{\xi}}\right)_{i}
$$

In (3.34) and (3.35), $\xi$ can be any subzones that are exposed to the open water region. Accordingly, $\vec{X}_{i}$ is on the exterior boundary of subzone $\xi$. In $(3.34), \vec{X}_{j}\left(j=1 \cdots \mathbb{N}_{\xi}\right)$ denotes the boundary points of subzone $\xi$ while $\vec{X}_{m}\left(m=1 \cdots \mathbb{N}_{S}\right)$ are on the boundary of open water (i.e. S), which includes the boundaries of all the forest patches. We should note that the outward normal direction along the boundary from the forest region is opposite to that from the open water region, i.e. $\hat{n}_{\xi}=-\hat{n}_{\mathrm{S}}$.

On the other hand, when $\vec{X}_{i}$ is along the interface between two subzones (i.e. $\vec{X}_{i} \in \mathrm{D}_{\xi \psi}$ ), combining (3.29) with (3.24) and (3.25) provides

$$
\frac{\Pi\left(\hat{k}_{\xi_{q}}\right)}{\cosh \hat{k}_{\xi_{q}} h}\left(\overline{\mathcal{G}}_{\xi_{q}}\right)_{i j}\left(\frac{\partial \mathcal{A}_{\xi_{q}}}{\partial \hat{n}_{\xi}}\right)_{j}=\sum_{r=0}^{\infty} \frac{\Gamma_{\psi_{r} \xi_{q}}}{\cosh \hat{k}_{\psi_{r}} h}\left(\overline{\mathcal{G}}_{\psi_{r}}\right)_{i k}\left(\frac{\partial \mathcal{A}_{\psi_{r}}}{\partial \hat{n}_{\psi}}\right)_{k}
$$

and

$$
\left(n_{\xi}+M_{\xi}\right) \sum_{q=0}^{\infty} \frac{\Gamma_{\xi_{q} \psi_{r}}}{\cosh \hat{k}_{\xi_{q}} h}\left(\frac{\partial \mathcal{A}_{\xi_{q}}}{\partial \hat{n}_{\xi}}\right)_{i}=-\left(n_{\psi}+M_{\psi}\right) \frac{\Pi\left(\hat{k}_{\psi_{r}}\right)}{\cosh \hat{k}_{\psi_{r}} h}\left(\frac{\partial \mathcal{A}_{\psi_{r}}}{\partial \hat{n}_{\psi}}\right)_{i}
$$

in which $\vec{X}_{j}\left(j=1 \cdots \mathbb{N}_{\xi}\right)$ and $\vec{X}_{k}\left(k=1 \cdots \mathbb{N}_{\psi}\right)$ represent the boundary points of subzones $\xi$ and $\psi$, respectively. Likewise, the outward normal directions along the interface with respect to these two adjacent subzones $(\xi$ and $\psi)$ are opposite, i.e. $\hat{n}_{\xi}=-\hat{n}_{\psi}$.

Equations (3.34), (3.35), (3.36) and (3.37) formulate a system of equations for solving the unknown $\mathcal{A}_{\xi_{q}}$, $\mathcal{A}_{\psi_{r}}$ and $\mathcal{B}_{p}$ as well as their normal derivatives along all the exterior forest boundaries and interfaces between subzones simultaneously. The wave solutions within each subzone and also the open water region can then be obtained by (3.2), (3.6), (3.8) and (3.12). It may be noted that the infinite $p, q$ and $r$ have to be truncated to finite numbers based on convergent tests.

Fig.2 is an example of a forest region, showing the sketch of discretization on both the exterior boundaries $\left(\mathrm{S}_{\xi}\right)$ and the interfaces $\left(\mathrm{D}_{\xi \psi}\right)$ of each subzone $\xi(\xi=1,2, \cdots, 9)$. For each subzone, the total number of boundary points are given as $\mathbb{N}_{\xi}$, e.g. Zone 3 has $\mathbb{N}_{3}$ elements along both the exterior boundary $\mathrm{S}_{3}$ and two interfaces $\mathrm{D}_{13} \& \mathrm{D}_{23}$. For open water, on the other hand, the boundary as noted has $\mathrm{S}=\sum \mathrm{S}_{\xi}=$ $\mathrm{S}_{1}+\mathrm{S}_{2}+\cdots+\mathrm{S}_{9}$. The interior angle and the element length are both indicated. The outward normal directions along the boundaries of subzones and of open water are presented as well. Note that for each subzone, the direction of integration along its boundary has to be counterclockwise as shown by dotted line with arrow in Fig.2. The corresponding numbering system of boundary elements for open water can be seen in Fig.S1 in the supplementary materials.

Based on the present approach, a computer progam named CHEWAVE-P (Cornell HomogEnization model on WAve-VEgetation interaction - Periodic waves) has been developed. The model has capability of solving the macro-scale wave propagations through a general forest region. The connection between microand macro-scale problems is also made. The iterative scheme for determining the bulk eddy viscosity for each subzone is included as well. To conduct the numerical computation with the present model, several inputs are required, such as incident wave conditions, forest properties, discretization of forest boundary and the desired computational domain. The outputs of the model include the corresponding drag coefficient, Reynolds number and eddy viscosity for each subzone. In addition, the dimensionless free surface elevation over the entire computational domain can be provided. A flow chart for running the numerical computation is shown in Fig.S2 and the details can be found in the supplementary materials. Note that the numerical results presented in this paper are all computed by this model. 


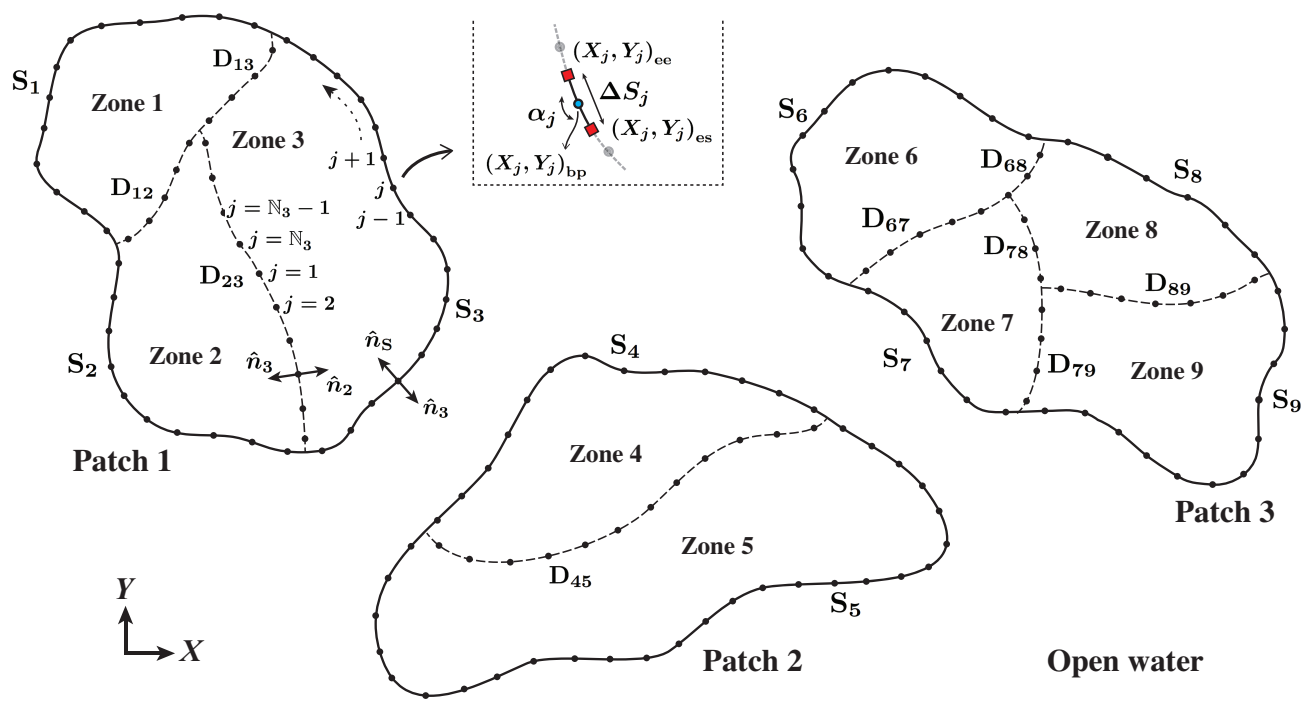

Figure 2: Discretization of boundary elements for a general forest region. For each subzone $\xi(=1 \cdots 9)$, the exterior boundary $\left(\mathrm{S}_{\xi}\right)$ as well as the interfaces $\left(\mathrm{D}_{\xi \psi}\right)$ are divided into elements where $\mathbb{N}_{\xi}$ gives the total number of its elements. $\left(X_{j}, Y_{j}\right)$ denotes the boundary points with subscripts bp, es and ee indicating the middle, starting and end point of $j_{\text {th }}$ element, respectively. The dotted line with arrow shows the direction of numbering system for each subzone (e.g. Zone 3 ). $\alpha_{j}$ shows the interior angle and $\Delta S_{j}$ represents the element length. $\hat{n}$ shows the outward normal directions to the boundaries of subzones and open water. Note that the number of dots in this figure only serves the purpose of illustration. A convergent test will be needed in the numerical computation to determine the resolution of boundary elements.

\section{Model validation and numerical results}

The circular forest studied in Liu et al. (2015) is first used to validate the present numerical model. With the homogenous forest configuration, a bulk eddy viscosity throughout the entire region is assumed. To this end, instead of iterating the bulk eddy viscosity, we simply use the values given by the semi-analytical theory. The comparisons between the present numerical results and the semi-analytical solutions by Liu et al. (2015) are excellent, showing negligible differences (e.g. Fig.5b). The detailed comparisons are therefore not shown here. To explore the present model further, the experiments on multiple circular forest patches (Maza 2015; Maza et al. 2016) are used as another special forest configuration. Numerical results and the comparisons with experimental data are presented herein.

\subsection{Waves scattered by multiple circular forest patches}

\subsubsection{Experimental setup}

To further test the present numerical model, one of Maza (2015)'s experiments with multiple patches is used as a special forest configuration. The experiments are conducted in the Directional Wave Basin at the University of Cantabria in Spain, which is the same facility for the single circular forest experiments (Liu et al. 2015). As shown in Fig.3, the basin is $24.1 \mathrm{~m}$ long, $8.6 \mathrm{~m}$ wide and $1.0 \mathrm{~m}$ deep and is equipped at one end with 10 paddles of piston-type wave maker. A gravel beach with a uniform slope $(\approx 1: 6.6)$ is used as passive wave absorber at the other end of the wave basin. The forest area, composed of four circular forest patches, is located around the middle of the wave basin. Each patch is $1.0 \mathrm{~m}$ in diameter and consists of 112 circular wooden cylinders with $3.0 \mathrm{~cm}$ in diameter. The arrangement of cylinders is the same as that applied to the circular forest experiments and can be referred to Fig.1.(b) in Liu et al. 2015. The cylinders are equally spaced $(\ell=9.0 \mathrm{~cm})$ with each square cell including one cylinder at the center. Each patch is treated as a homogeneous forest region without subzones. The porosity is around $91.27 \%$. Totally 29 wave gauges are used to record the water surface displacement inside and outside of the patches. Only parts 
of wave gauges are shown in Fig.3. The experimental conditions are listed in Table 1. The incident wave conditions are estimated based on the time records of wave gauges 1, 2 and 3 (Isaacson 1991).

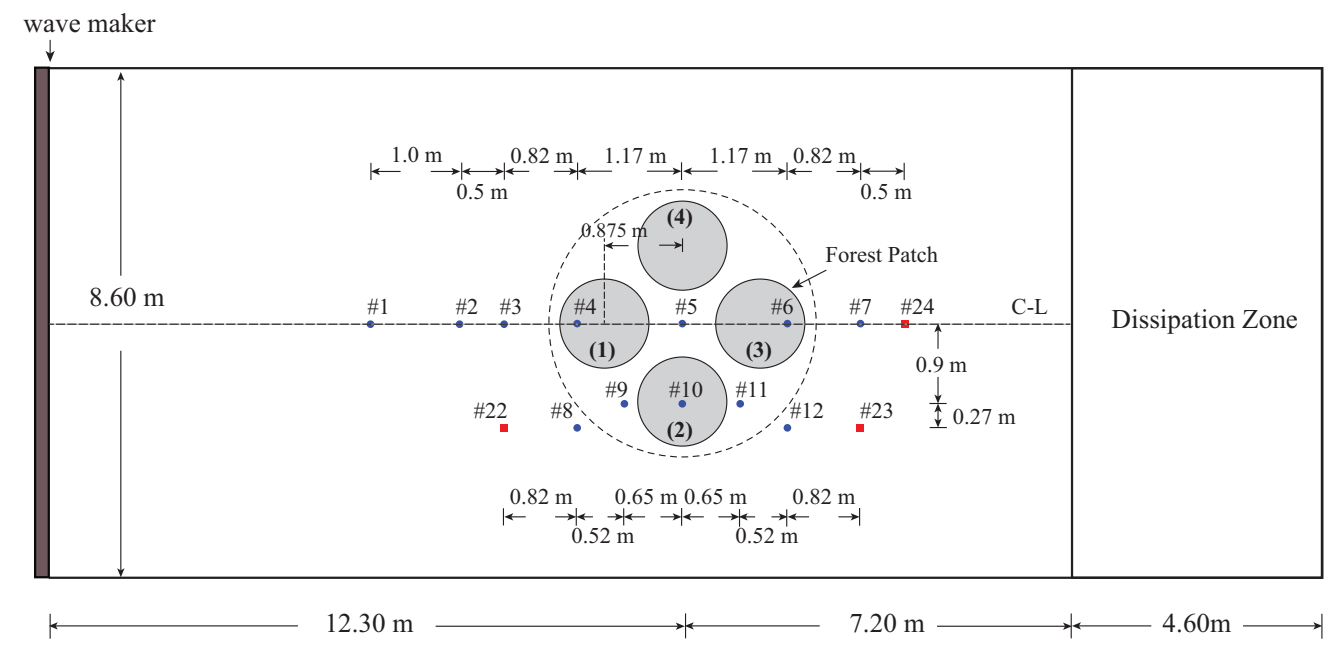

(a) Top View

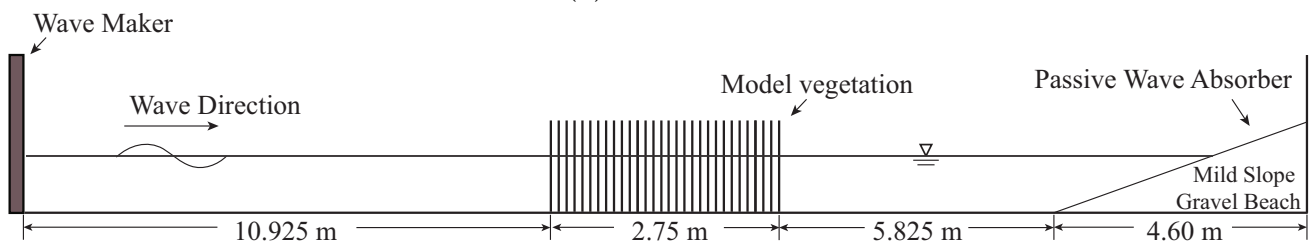

(b) Side view

Figure 3: Test layout of multiple circular forest patches used in Maza (2015)'s experiments. Each patch is $1.0 \mathrm{~m}$ in diameter. The bigger dashed circle indicates the single circular forest in Liu et al. (2015), which has $3.0 \mathrm{~m}$ in diameter. Parts of the wave gauges used in the experiments are presented and numbered with dots and squares representing the resistive-type and acoustic-type gauges. The patches are also numbered, i.e. $(\xi), \xi=1-4$. C-L denotes the centerline $(Y=0)$. More details of the experimental setup can be referred to Maza (2015)'s Fig.8.2 and Table 8.1.

Table 1: Experimental conditions and corresponding dimensionless parameters in Maza (2015)'s experiments.

\begin{tabular}{c|c|cccccc}
\hline Case & $h(\mathrm{~cm})$ & $T(\mathrm{~s})$ & $H_{\text {inc }}(\mathrm{cm})$ & $L(\mathrm{~m})$ & $k h$ & $k A$ & $\sigma_{\text {cir }}$ \\
\hline 1 & & 1.50 & 5.34 & 2.3421 & 0.8048 & 0.0716 & $2.4481 \mathrm{E}-03$ \\
2 & & 2.00 & 4.65 & 3.2578 & 0.5786 & 0.0448 & $4.2983 \mathrm{E}-03$ \\
3 & \multirow{3}{*}{30} & 2.50 & 4.45 & 4.1500 & 0.4542 & 0.0337 & $5.7201 \mathrm{E}-03$ \\
4 & & 3.00 & 5.17 & 5.0000 & 0.3770 & 0.0325 & $7.4942 \mathrm{E}-03$ \\
5 & & 1.50 & 11.60 & 2.3421 & 0.8048 & 0.1556 & $5.9737 \mathrm{E}-03$ \\
6 & & 2.00 & 9.95 & 3.2578 & 0.5786 & 0.0960 & $8.5060 \mathrm{E}-03$ \\
\hline
\end{tabular}

The above $h, T$ and $L$ represent the constant water depth, the incident wave period and wavelength, respectively. The incident wave height $H_{\text {inc }}$ is obtained from the measurements at wave gauges 1,2 and $3 . k$ denotes the incident wavenumber. The cell porosity is approximately $91.27 \%$. $\sigma_{\text {cir }}$ gives the bulk eddy viscosity given by the semi-analytical theory for one single circular forest (Liu et al. 2015).

\subsubsection{Numerical results}

We consider each circular patch as a homogeneous subzone of the entire forest region. Within each patch, the eddy viscosity is assumed as a bulk value but can be different from each other, i.e. $\sigma_{\xi}$ with $\xi=1 \cdots 4$ 
denoting the indices of patches in Fig.3. Thus, the cell problem for each patch has to be solved independently, yielding the corresponding values of the complex coefficients $M_{\xi}, N_{\xi}$ and the wavenumber $\hat{k}_{\xi_{q}}$. For each patch (i.e. patch $\xi$ ), the outward normal direction of the boundary can be represented by $r_{\xi}$ as sketched in Fig.4 (i.e. $r_{\xi} \equiv \hat{n}_{\xi}=-\hat{n}_{\mathrm{S}}$ ). The matching conditions in (3.34) and (3.35) can then be modified as:

$$
\frac{\Pi\left(\hat{k}_{\xi_{q}}\right)}{\cosh \hat{k}_{\xi_{q}} h}\left(\mathcal{G}_{\xi_{q}}\right)_{i j}\left(\frac{\partial \mathcal{A}_{\xi_{q}}}{\partial r_{\xi}}\right)_{j}=A_{0} \frac{\Gamma_{0 \xi_{q}}}{\cosh k_{0} h}\left(e^{\mathrm{i} k_{0} X_{1}}\right)_{i}-\sum_{p=0}^{\infty} \frac{\Gamma_{p \xi_{q}}}{\cosh k_{p} h}\left(\mathcal{Q}_{p}\right)_{i m}\left(\frac{\partial \mathcal{B}_{p}}{\partial r_{\xi}}\right)_{m}
$$

and

$$
\left(n_{\xi}+M_{\xi}\right) \sum_{q=0}^{\infty} \frac{\Gamma_{\xi_{q} p}}{\cosh \hat{k}_{\xi_{q}} h}\left(\frac{\partial \mathcal{A}_{\xi_{q}}}{\partial r_{\xi}}\right)_{i}=A_{0} \delta_{0 p} \frac{\Pi\left(k_{0}\right)}{\cosh k_{0} h}\left(\frac{\partial e^{\mathrm{i} k_{0} X_{1}}}{\partial r_{\xi}}\right)_{i}+\frac{\Pi\left(k_{p}\right)}{\cosh k_{p} h}\left(\frac{\partial \mathcal{B}_{p}}{\partial r_{\xi}}\right)_{i}
$$

in which $i=1 \cdots \mathbb{N}_{\xi}, j=1 \cdots \mathbb{N}_{\xi}$ and $m=1 \cdots \mathbb{N}_{\mathrm{S}}$ with $\mathbb{N}_{\mathrm{S}}=\sum_{\xi=1}^{4} \mathbb{N}_{\xi}$. Accordingly, a system of equations is formulated for solving $\partial \mathcal{A}_{\xi_{q}} / \partial r_{\xi}$ and $\partial \mathcal{B}_{p} / \partial r_{\xi}$.

To conduct the numerical computations, the discretization of forest boundary has to be done beforehand as shown in Fig.4. Different numbers of boundary elements are examined as a convergence test. Comparing with the semi-analytical solutions for a circular forest (Liu et al. 2015), the mean error (\%) of the numerical results over the computational domain for different discretization resolutions are presented in Fig.5a. A part of the dimensionless wave amplitude profiles along the centerline is shown in Fig.5b. It can be observed that the numerical results approach the semi-analytical solutions when the number of boundary elements is increased, i.e. the central angle is decreased. Therefore, following the convergence test and requiring the mean error lower than $10^{-1} \%$, the number of the boundary elements for each patch is set as 180 (i.e. $\left.\mathbb{N}_{\xi}=180, \xi=1-4\right)$ with $\pi / 90 \mathrm{rad}$ as the central angle. Totally 720 elements (i.e. $\mathbb{N}_{\mathrm{S}}=720$ ) are used to discretize the boundary of four circular patches. Uniform solution along each element is assumed. Once the unknown functions $\mathcal{A}_{\xi_{q}}$ and $\mathcal{B}_{p}$ along the boundaries are solved, the wave solutions within each patch as well as the open water region can be obtained by (3.2), (3.6), (3.8) and (3.12).

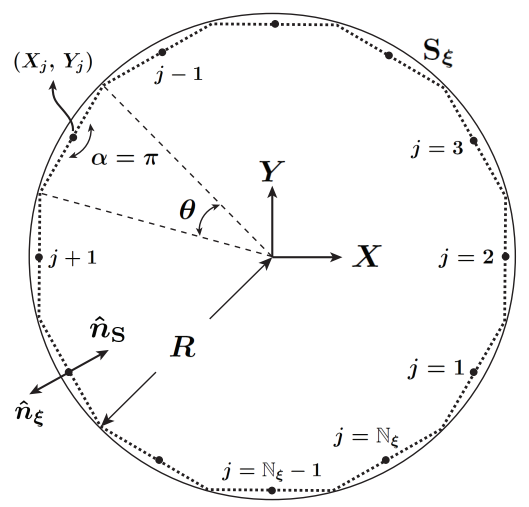

Figure 4: Sketch of the discretization of boundary elements (dotted lines) for a homogeneous circular forest patch. A single bulk eddy viscosity and drag coefficient is yielded within each patch. Point $\left(X_{j}, Y_{j}\right)$ denotes the middle point of $j_{\text {th }}$ element with interior angle $\alpha=\pi$ for smooth element. $\mathrm{S}_{\xi}$ denotes the boundary of patch $\xi$ with $\hat{n}_{\xi}$ and $\hat{n}_{\mathrm{S}}$ showing the outward normal directions from the forest side and the open water region, respectively. In numerical computation the total number of elements $\mathbb{N}_{\xi}$ for one patch is set as 180 with $\theta=2^{\circ}$. 


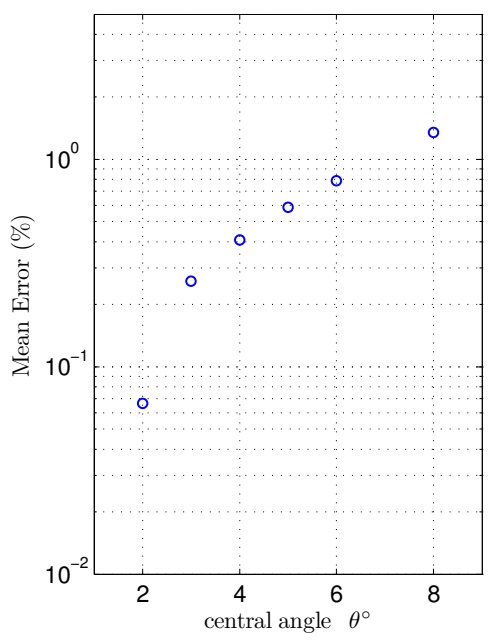

(a)

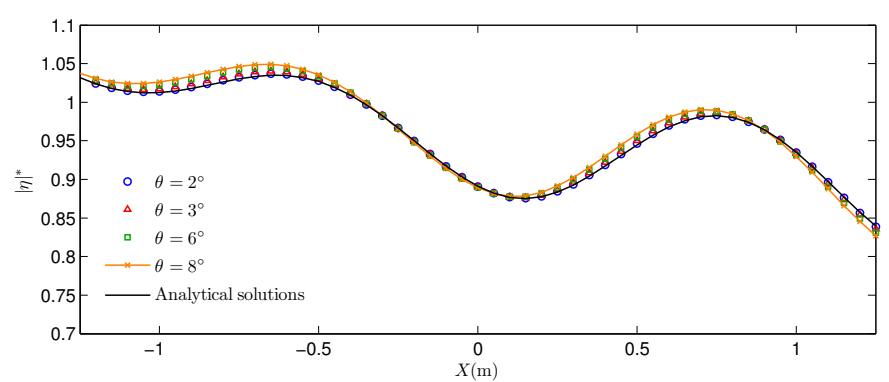

(b)

Figure 5: Comparison of numerical results for a circular forest by using different resolutions of boundary elements: (a) shows the mean relative error (\%) comparing with the semi-analytical solution (Liu et al. 2015); (b) shows the dimensionless wave amplitude along the centerline obtained by different discretization.

As mentioned above, each patch is considered as a subzone of the entire forest region and should have its own bulk value of eddy viscosity: $\sigma_{\xi}$ with $\xi=1 \cdots 4$. Each eddy viscosity can be determined respectively by the energy concept and the iterative scheme introduced in Liu et al. (2015). Before iterating the eddy viscosity, we first employ the bulk value given by the semi-analytical theory for one single circular forest (Liu et al. 2015), assigning the value to all the four patches to observe the effects of this special forest configuration on the incoming wave attenuation. Note that the details of estimation on the eddy viscosity and the associated iterative scheme can be found in Liu et al. (2015). Totally 6 cases in Maza (2015)'s experiments are tested and the wave conditions are in Table 1 . The comparisons between the model predictions and the experimental data for two forest configurations are presented in Fig.6 - 9. The corresponding wave gauges can be found in Fig.3. In the results, the reduced wave amplitudes due to the energy dissipation inside the forest patches are observed. However, due to the arrangement of wave gauges, it does not allow the detailed comparison. Overall, the agreement over the entire forest region is reasonable. The present model also works well even for the cases with higher nonlinearity (e.g. Case 5 \& 6). In Fig.6 and 7, the effects of incident wave height on wave attenuation are also compared. As presented, larger waves (Case $5 \& 6$ ) have higher normalized dissipation rates in comparison with smaller waves (Case $1 \& 2$ ). The comparison of longer waves with reasonable trends is also presented in Fig.8 and 9. As shown in Fig.3, more cylinders are in use for the single circular forest comparing with four smaller patches in the vegetated area. Thus, it can be observed that the former is more efficient on dissipating the incoming wave energy than the latter. The refracted waves converged behind the forest region are observed for both configurations, resulting in slightly increasing wave amplitude. While the circular forest is more efficient for damping shorter waves (Liu et al. 2015), it is not clear for multiple patches.

In the present model, a sharp contrast between the wavelength and the tree spacing is required. The leading-order free surface elevation is independent of the micro-scale coordinates and only varies with the macro-scale coordinates. The free surface fluctuation can be captured by the solutions of $\mathcal{O}(\varepsilon)$. Ideally, the macro-scale grid size (i.e. $\Delta X_{i}$ ) in the numerical computations shall be greater than the cylinder spacing (i.e. $\ell$ ) such that a unit cell in the micro-scale computation can be an averaged representative of several real cylinders. However, due to the limitations on laboratory experiments, the current cylinder spacing and the computational grid size are in the same order of magnitude, i.e. $\mathcal{O}(\ell) \approx \mathcal{O}\left(\Delta X_{i}\right)$. Therefore, a uniform leading-order free surface within a cell in real scale is used to compare with the gauge measurements. In reality, the free surface elevation would vary around cylinders and the wave gauge can only provide instantaneous measurements at a specific location. This might contribute to the discrepancies between 
numerical results and the experimental data. Beside, it could be observed that the wave dissipation becomes lower when the wavelength is larger. This might bring about difficulties on interpreting the accuracy of experimental data. For example, along $Y=-0.9 \mathrm{~m}$ in Fig.8 and 9, the measured wave amplitude is increasing when propagating through the forest. Also, the wavelength of case 3 and 4 becomes 4 and 5 times of the smaller patch size. As mentioned above, it is not allowed to compare the wave damping trends in numerical results with measurements due to the lack of available gauges inside each patch.

The snapshots of dimensionless free surface elevation over forest patches as well as the open water region for Case 1 are presented in Fig.10. Although the incident wavelength is more than twice of the patch size, we can still recognize the refraction caused by the forest region. Note that the numerical results presented above are based on the bulk eddy viscosity for one single circular forest. However, the eddy viscosity should be different in different patches. To investigate the possible influences of different values of eddy viscosity on the prediction of wave attenuation, Case 1 (in Table 1) is used to perform the iterative scheme, which requires that the relative error of the bulk eddy viscosity $\left(\sigma_{\xi}\right)$ within each patch from two successive iterations has to be sufficiently small (e.g. $\leq 10^{-3}$ ). The converged values can usually be reached within 10-20 iterations (Liu et al. 2015). As a result, the iteration yields the dimensionless eddy viscosity for four patches as $\left[\sigma_{1}, \sigma_{2}, \sigma_{3}, \sigma_{4}\right]=[2.4284,2.3701,2.2554,2.3701]\left(\times 10^{-3}\right)$. Insignificant difference (below $0.5 \%$ ) is observed. It may be noted that the distance between patches and the patch size comparing with the single circular forest may affect the estimation of eddy viscosity and the resultant wave attenuation.
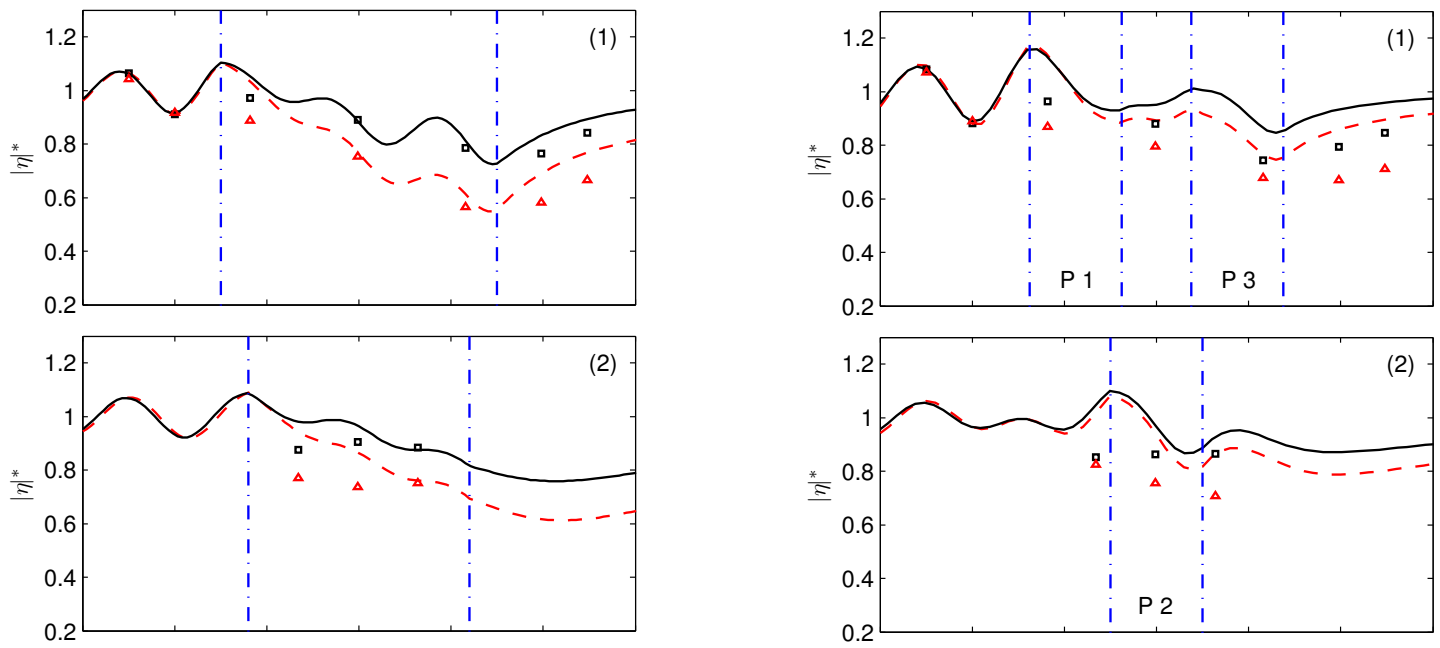

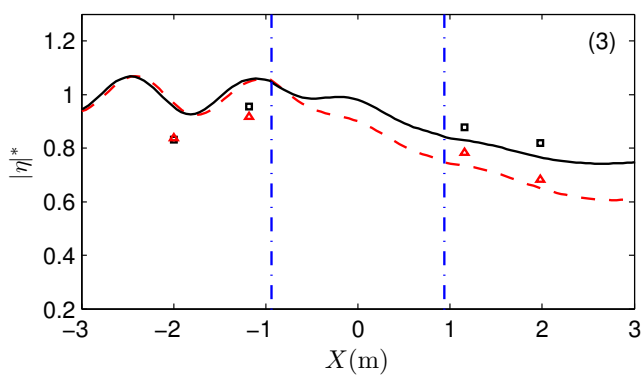

(a) Circular forest

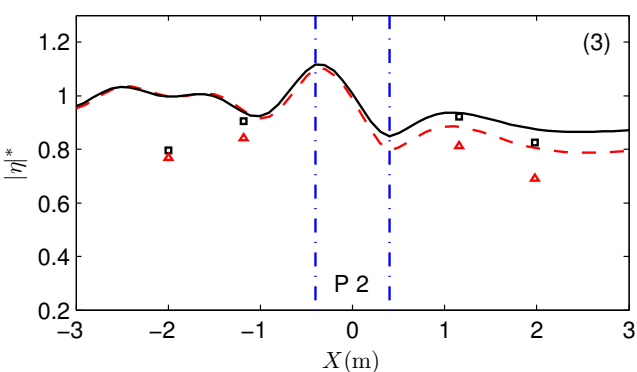

(b) Multiple forest patches

Figure 6: Spatial variation of dimensionless wave amplitude I - along (1) $Y=0 \mathrm{~m}$ (WG2, 3, 4, 5, 6, 7, 24), (2) $Y=-0.9 \mathrm{~m}$ (WG9, 10, 11) and (3) $Y=-1.17 \mathrm{~m}$ (WG22, 8, 12, 23). Two wave heights are compared: Case 1 - solid lines (model predictions) and squares (gauge data); Case 5 - dashed lines (model predictions) and triangles (gauge data). Dot-dashed vertical lines show the forest edges. $\mathrm{P}_{\xi}(\xi=1-4)$ denotes the patch index. 

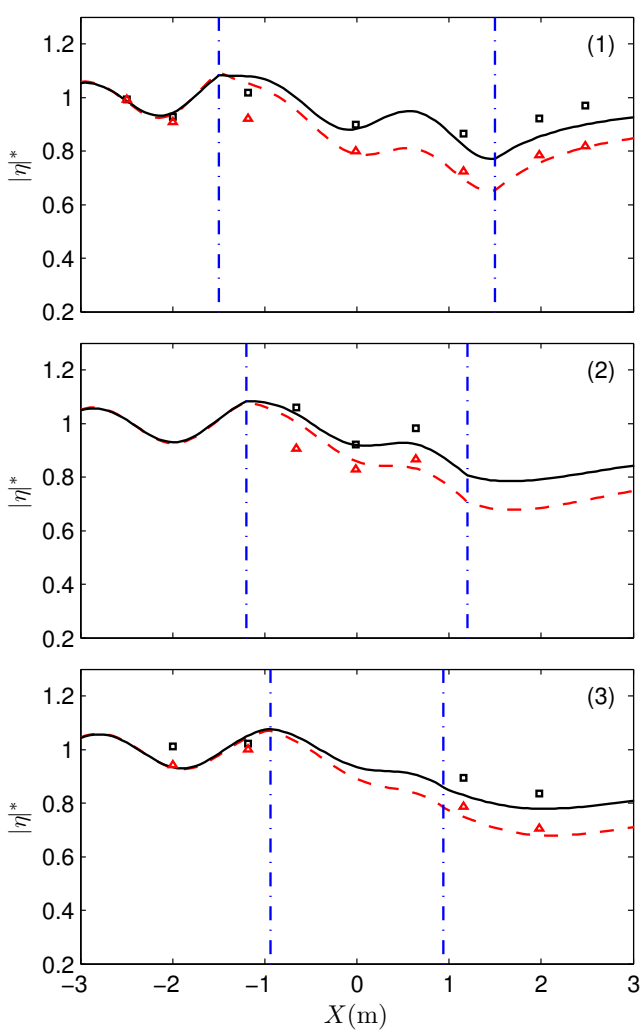

(a) Circular forest
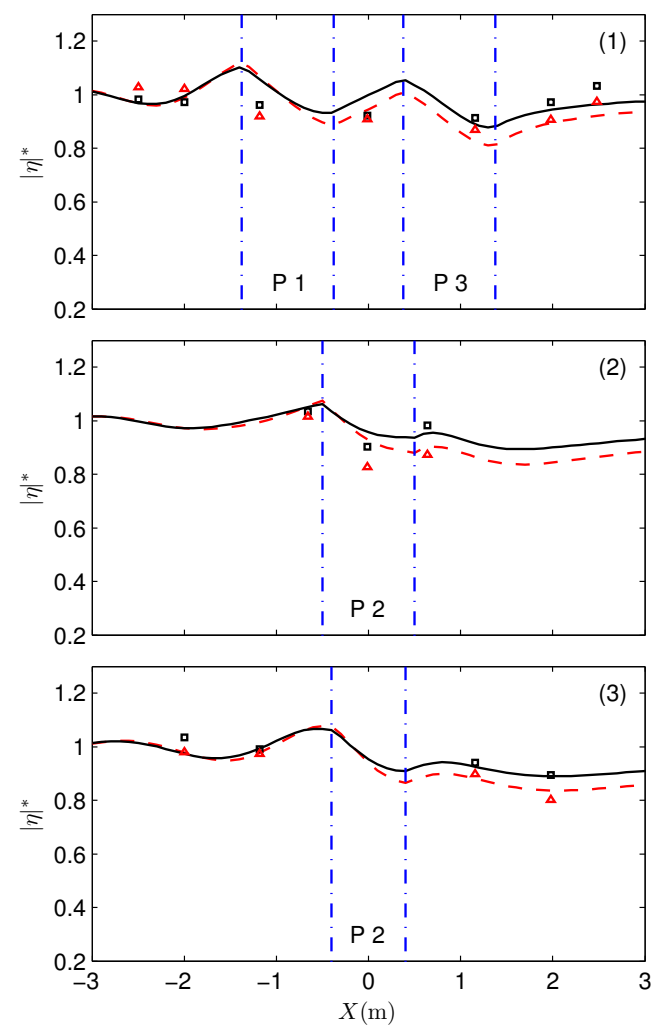

(b) Multiple forest patches

Figure 7: Spatial variation of dimensionless wave amplitude II - along (1) $Y=0 \mathrm{~m}$ (WG2, 3, 4, 5, 6, 7, 24), (2) $Y=-0.9 \mathrm{~m}$ (WG9, 10, 11) and (3) $Y=-1.17 \mathrm{~m}$ (WG22, 8, 12, 23). Two wave heights are compared: Case 2 - solid lines (model predictions) and squares (gauge data); Case 6 - dashed lines (model predictions) and triangles (gauge data). Dot-dashed vertical lines show the forest edges. $\mathrm{P}_{\xi}(\xi=1-4)$ denotes the patch index. 

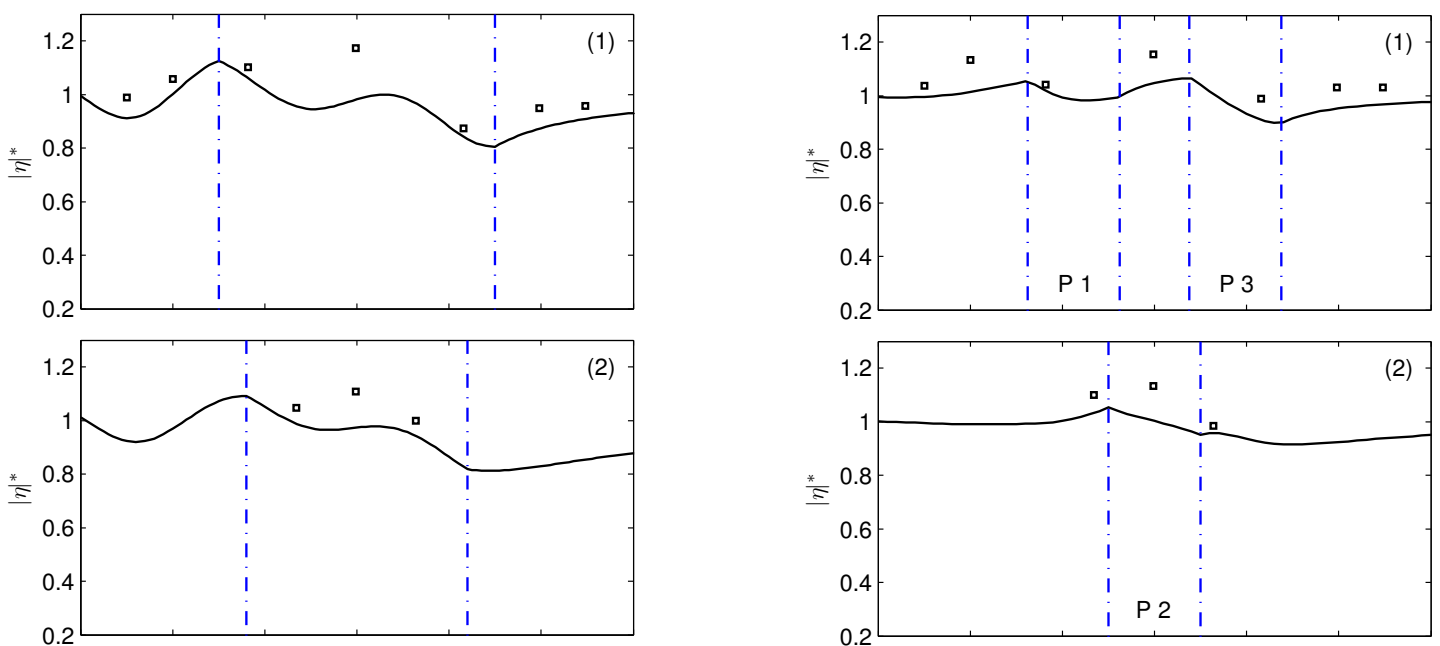

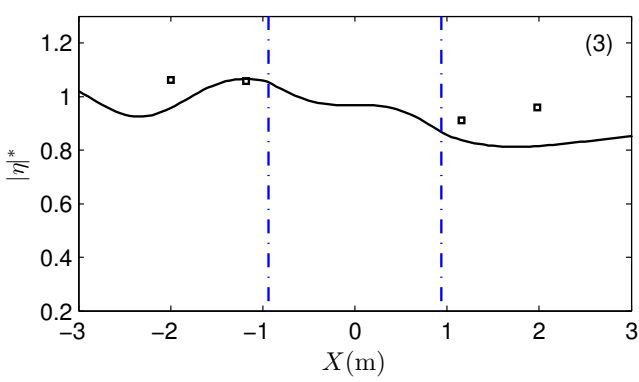

(a) Circular forest

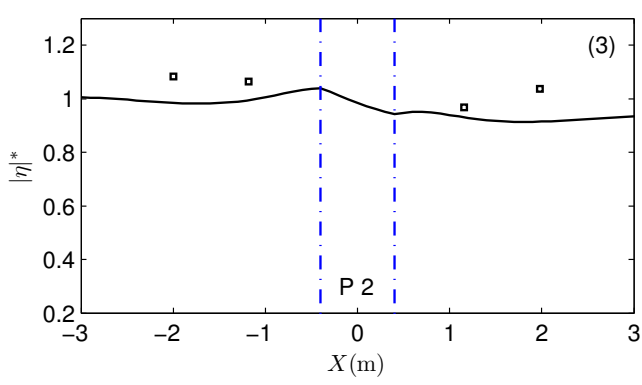

(b) Multiple forest patches

Figure 8: Spatial variation of dimensionless wave amplitude III - along (1) $Y=0 \mathrm{~m}$ (WG2, 3, 4, 5, 6, 7, 24), (2) $Y=-0.9 \mathrm{~m}$ (WG9, 10, 11) and (3) $Y=-1.17 \mathrm{~m}$ (WG22, 8, 12, 23). Case 3 - solid lines (model predictions) and squares (gauge data). Dot-dashed vertical lines show the forest edges. $\mathrm{P}_{\xi}(\xi=1-4)$ denotes the patch index. 

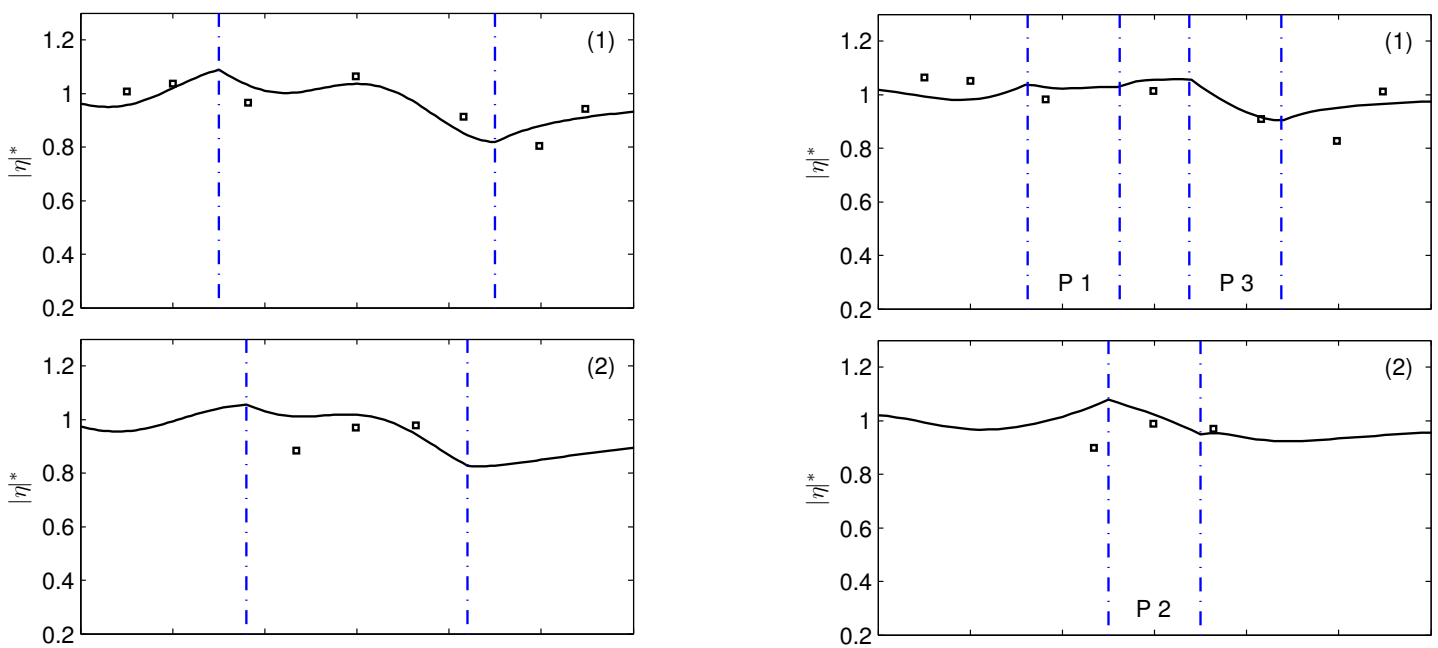

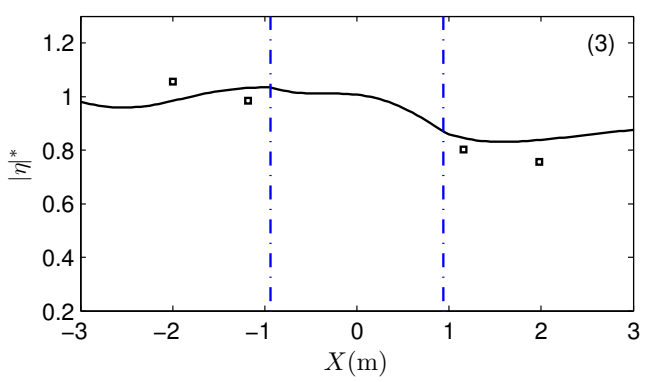

(a) Circular Forest

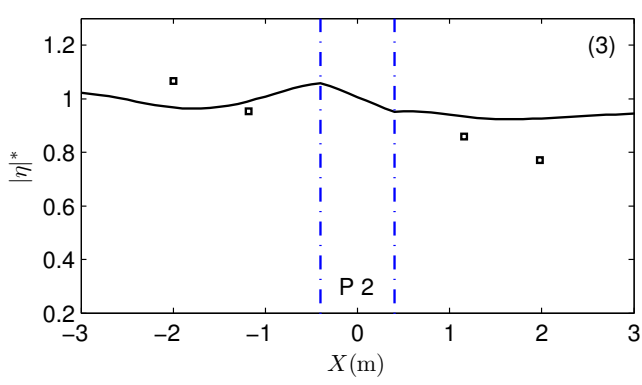

(b) Multiple forest patches

Figure 9: Spatial variation of dimensionless wave amplitude IIII - along (1) $Y=0 \mathrm{~m}$ (WG2, 3, 4, 5, 6, 7, 24), (2) $Y=-0.9 \mathrm{~m}$ (WG9, 10, 11) and (3) $Y=-1.17 \mathrm{~m}$ (WG22, 8, 12, 23). Case 4 - solid lines (model predictions) and squares (gauge data). Dot-dashed vertical lines show the forest edges. $\mathrm{P}_{\xi}(\xi=1-4)$ denotes the patch index. 


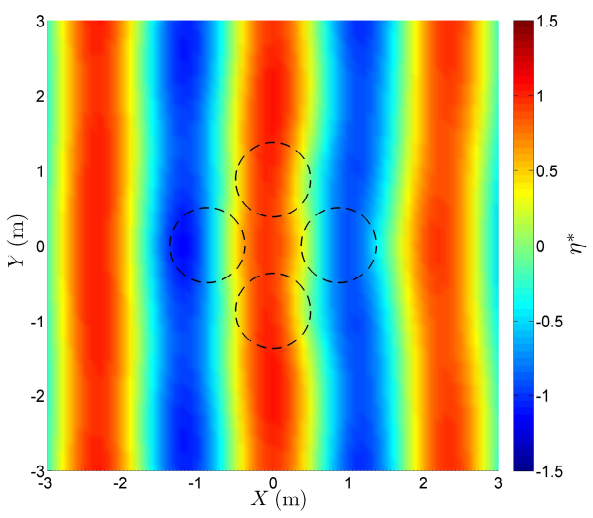

(a) $t=0(\mathrm{~s})$

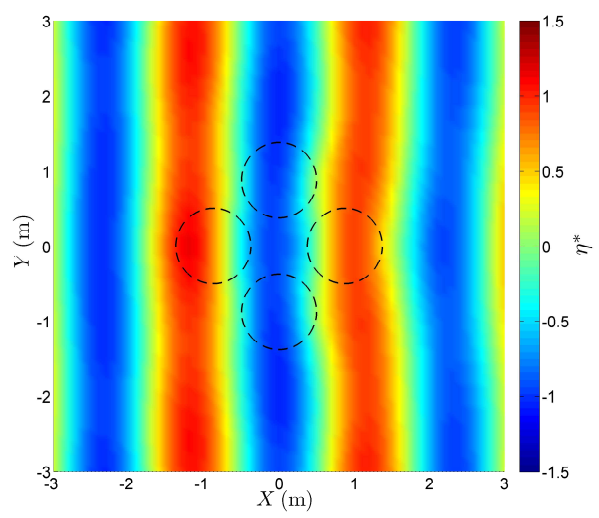

(c) $t=T / 2(\mathrm{~s})$

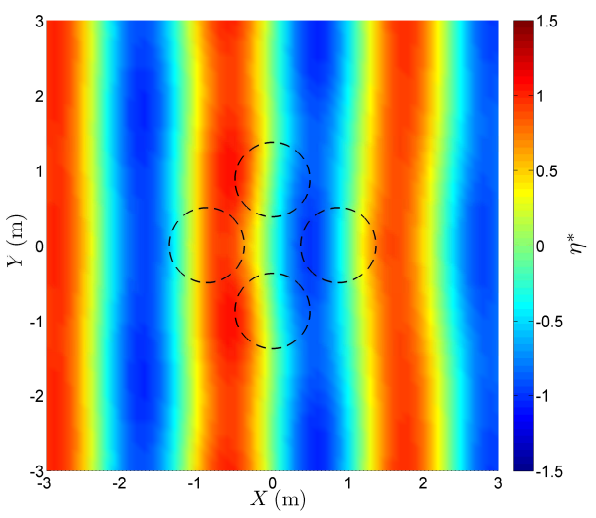

(b) $t=T / 4(\mathrm{~s})$

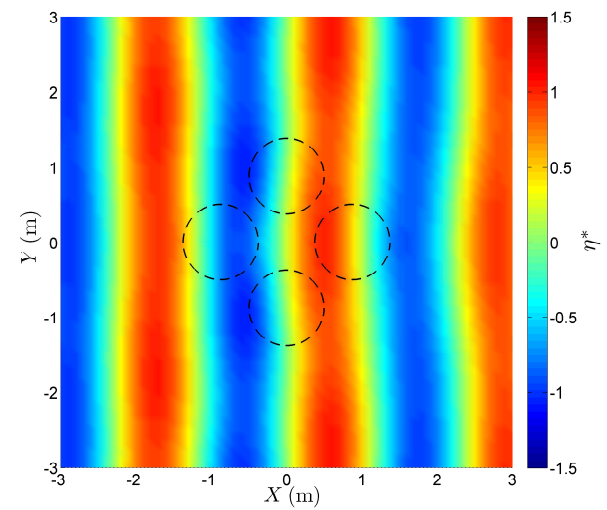

(d) $t=3 T / 4(\mathrm{~s})$

Figure 10: Snapshots of the dimensionless free surface elevation over the outside and inside forest region at different times. The incident wave condition has $T=1.50 \mathrm{~s}$ and $H_{\text {inc }}=5.34 \mathrm{~cm}$ with constant water depth $h=30 \mathrm{~cm}$. The dashed line shows the boundary of the forest patches.

\subsubsection{Model demonstration}

To demonstrate the capability of the present model, here we select two different non-circular forest configurations. Applying the same cylinder arrangement and cell porosity as used for multiple circular patches (i.e. Sec.4.1.1), the dissipation of wave amplitude through a rectangular forest (Fig.11) and two separated rectangular patches (Fig.12) can both be observed. In addition, the refraction of propagating waves for these two forest configurations is being captured by the present model. Note again that the discretization of forest boundary is required to be prepared before preforming the computations. The corresponding animations showing the dimensionless free surface elevation through different forest configurations (e.g. Fig.10 - 12) can be found in the supplementary materials. 


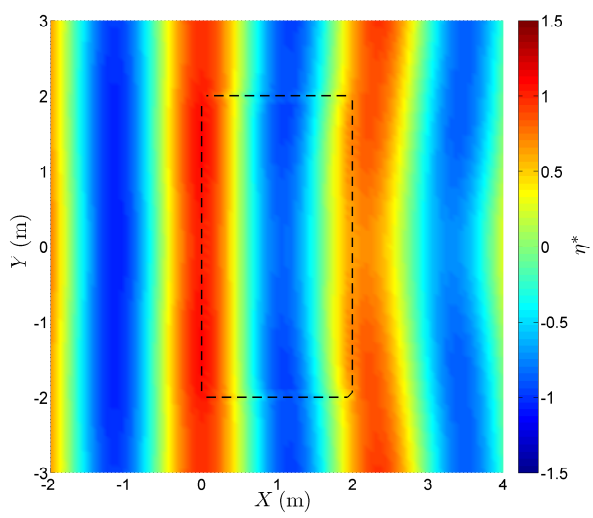

(a) $t=0(\mathrm{~s})$

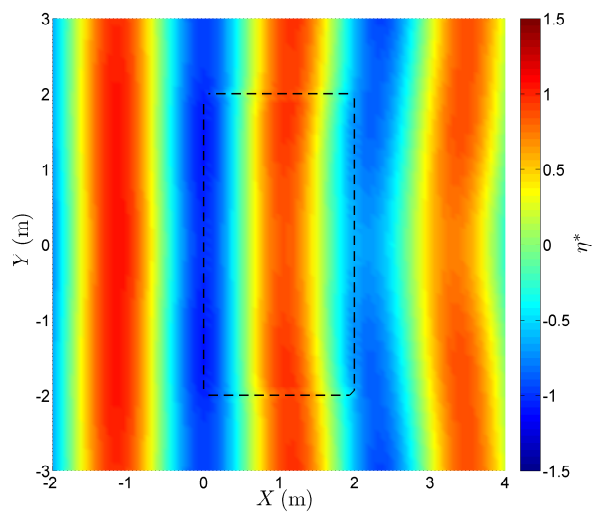

(c) $t=T / 2(\mathrm{~s})$

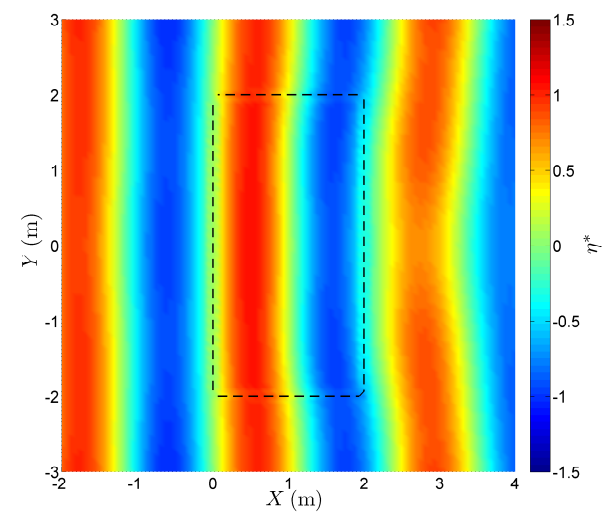

(b) $t=T / 4(\mathrm{~s})$

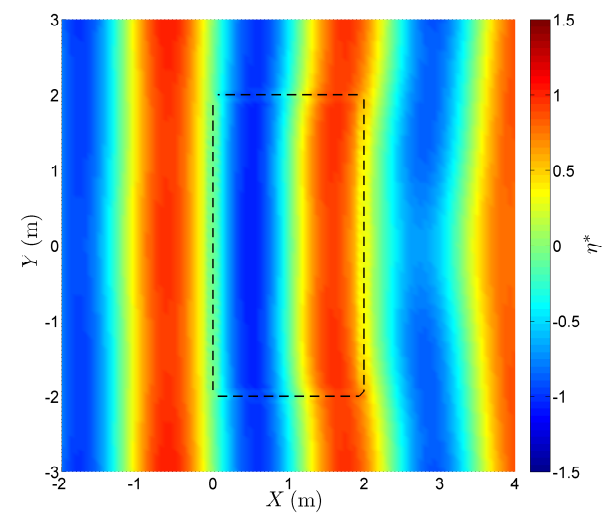

(d) $t=3 T / 4(\mathrm{~s})$

Figure 11: Snapshots of dimensionless free surface elevation over one rectangular forest within one wave period. The incident wave condition has $T=1.50 \mathrm{~s}$ and $H_{\text {inc }}=5.34 \mathrm{~cm}$ with constant water depth $h=30$ $\mathrm{cm}$. The dashed line shows forest boundary. 


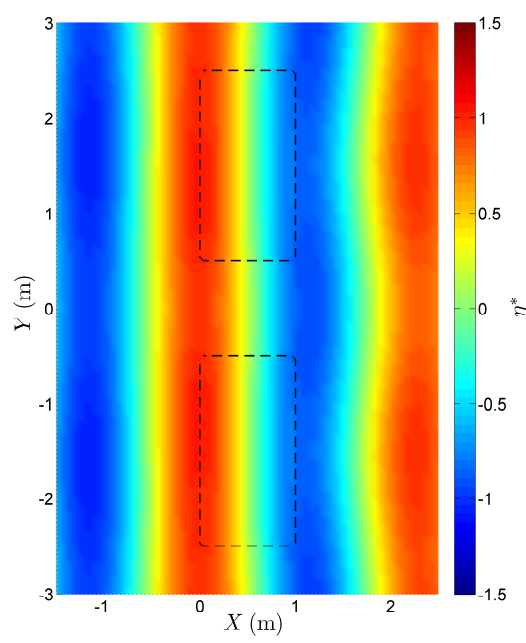

(a) $t=0(\mathrm{~s})$

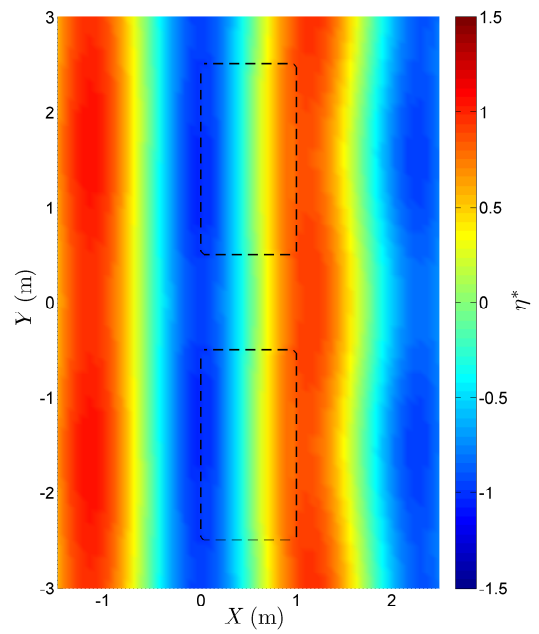

(c) $t=T / 2(\mathrm{~s})$

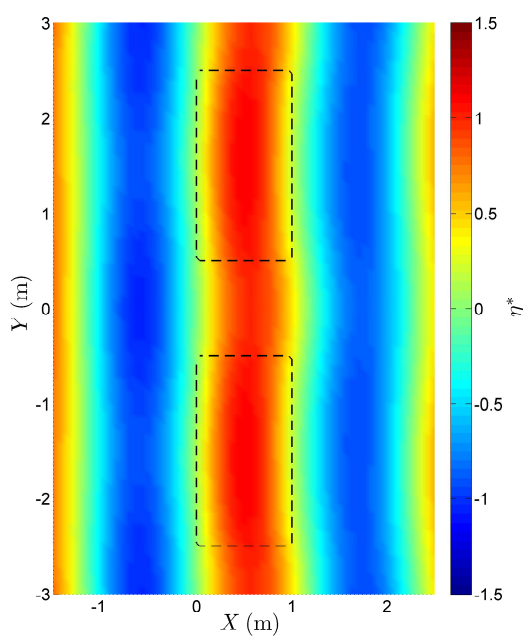

(b) $t=T / 4(\mathrm{~s})$

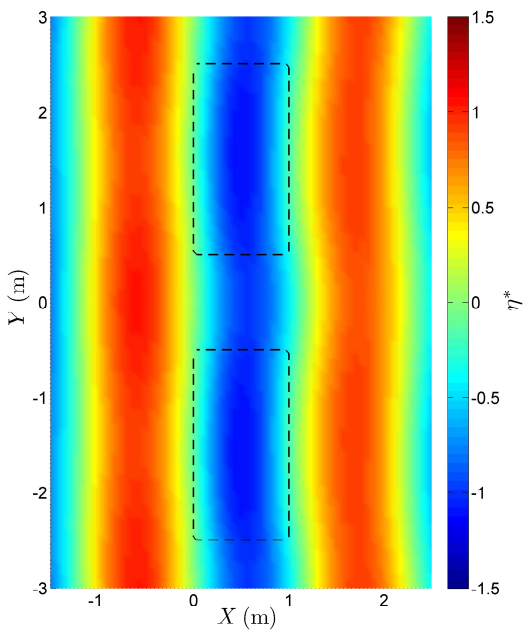

(d) $t=3 T / 4(\mathrm{~s})$

Figure 12: Snapshots of dimensionless free surface elevation over two rectangular patches within one wave period. The incident wave condition has $T=1.50 \mathrm{~s}$ and $H_{\text {inc }}=5.34 \mathrm{~cm}$ with constant water depth $h=30$ $\mathrm{cm}$. The dashed line shows the boundary of the forest patches.

In addition to multiple patches, a single circular region with two subzones as sketched in Fig.13 is used to demonstrate the numerical results. The semi-analytical solutions can be obtained as shown in Liu et al. (2015). For the open water region, the solution form is as

$$
\begin{aligned}
\phi_{\mathrm{S}} & =A_{0} \sum_{m=0}^{\infty} \epsilon_{m} \mathrm{i}^{m} \cos m \theta\left\{\left[J_{m}\left(k_{0} r\right)+C_{m 0} H_{m}^{(1)}\left(k_{0} r\right)\right] \frac{\cosh k_{0}(Z+h)}{\cosh k_{0} h}\right\} \\
& +A_{0} \sum_{m=0}^{\infty} \epsilon_{m} \mathrm{i}^{m} \cos m \theta\left[\sum_{p=1}^{\infty} C_{m p} K_{m}\left(\kappa_{p} r\right) \frac{\cos \kappa_{p}(Z+h)}{\cos \kappa_{p} h}\right], \quad r>R
\end{aligned}
$$

in which $J_{m}, H_{m}^{(1)}$ and $K_{m}$ represent respectively the Bessel function of the first kind, the Hankel function of the first kind and the modified Bessel function of second kind. $\epsilon_{m}$ is the Jacobi symbol, defined as $\epsilon_{0}=1$ and $\epsilon_{m}=2$ when $m \geq 1$. Note that $k_{p}=\mathrm{i} \kappa_{p}$ (when $p \geq 1$ ). For two subzones, the solutions are

$$
\phi_{1}=A_{0} \sum_{m=0}^{\infty} \epsilon_{m} \mathrm{i}^{m} \cos m \theta\left\{\sum_{q=0}^{\infty}\left[B_{m q} J_{m}\left(\gamma_{1} \hat{k}_{1 q} r\right)+B_{m q}^{\prime} Y_{m}\left(\gamma_{1} \hat{k}_{1 q} r\right)\right] \frac{\cosh \hat{k}_{1 q}(Z+h)}{\cosh \hat{k}_{1 q} h}\right\}, \quad R_{2}<r<R
$$


and

$$
\phi_{2}=A_{0} \sum_{m=0}^{\infty} \epsilon_{m} \mathrm{i}^{m} \cos m \theta\left[\sum_{e=0}^{\infty} D_{m e} J_{m}\left(\gamma_{2} \hat{k}_{2 e} r\right) \frac{\cosh \hat{k}_{2 e}(Z+h)}{\cosh \hat{k}_{2 e} h}\right], \quad r<R_{2}
$$

where $Y_{m}$ denotes the Bessel function of the second kind. $\hat{k}_{1 q}$ and $\hat{k}_{2 e}$ satisfy their dispersion relationships, respectively, i.e.

$$
1=\left(\frac{n_{1}+N_{1}}{n_{1}}\right) \hat{k}_{1 q} \tanh \hat{k}_{1 q} h \quad \text { and } \quad 1=\left(\frac{n_{2}+N_{2}}{n_{2}}\right) \hat{k}_{2 e} \tanh \hat{k}_{2 e} h
$$

The matching conditions along the exterior boundary (i.e. $r=R$ ) are in (3.13) and (3.15) while the conditions along the interface between two subzones (i.e. $\left.r=R_{2}\right)$ are in (3.20) and (3.22). With the application of orthogonality as presented in Liu et al. (2015)'s (A.3), (A.4), (A.8) and (A.9), a system of algebraic equations can be obtained for solving the unknown coefficients $C_{m 0}, C_{m p}, B_{m q}, B_{m q}^{\prime}$ and $D_{m e}$ :

$$
[\mathbb{A}]\{\mathbb{C}\}=\{\mathbb{R H} \mathbb{S}\}
$$

in which

$$
\mathbb{C}=\left\{\begin{array}{c}
C_{m 0} \\
C_{m p} \\
B_{m q} \\
B_{m q}^{\prime} \\
D_{m e}
\end{array}\right\} \text { and } \quad \mathbb{R} \mathbb{H} \mathbb{S}=\left\{\begin{array}{c}
-J_{m}\left(k_{0} R\right) \frac{\Gamma_{01}}{\cosh k_{0} h} \\
\mathbf{0} \\
j_{m}\left(k_{0} R\right) \frac{\Pi\left(k_{0}\right)}{\cosh k_{0} h} \\
\mathbf{0} \\
\mathbf{0}
\end{array}\right\} .
$$

The nonzero elements of the coefficient matrix $\mathbb{A}$ are

$$
\begin{aligned}
& \mathbb{A}_{11}=H_{m}^{(1)}\left(k_{0} R\right) \frac{\Gamma_{01_{q}}}{\cosh k_{0} h}, \quad \mathbb{A}_{12}=\sum_{p=1}^{n_{p}} K_{m}\left(\kappa_{p} R\right) \frac{\Gamma_{p 1_{q}}}{\cos \kappa_{p} h}, \quad \mathbb{A}_{13}=-J_{m}\left(\gamma_{1} \hat{k}_{1_{q}} R\right) \frac{\Pi\left(\hat{k}_{1_{q}}\right)}{\cosh \hat{k}_{1_{q}} h}, \quad \mathbb{A}_{14}=-Y_{m}\left(\gamma_{1} \hat{k}_{1_{q}} R\right) \frac{\Pi\left(\hat{k}_{1_{q}}\right)}{\cosh \hat{k}_{1_{q}} h}, \\
& \mathbb{A}_{22}=-\dot{K}_{m}\left(\kappa_{p} R\right) \frac{\Pi\left(\kappa_{p}\right)}{\cos \kappa_{p} h}, \quad \mathbb{A}_{23}=M_{1}^{\prime} \sum_{q=0}^{n_{q}} \dot{J}_{m}\left(\gamma_{1} \hat{k}_{1_{q}} R\right) \frac{\Gamma_{p 1_{q}}}{\cosh \hat{k}_{1_{q}} h}, \quad \mathbb{A}_{24}=M_{1}^{\prime} \sum_{q=0}^{n_{q}} \dot{Y}_{m}\left(\gamma_{1} \hat{k}_{1_{q}} R\right) \frac{\Gamma_{p 1_{q}}}{\cosh \hat{k}_{1_{q}} h}, \\
& \mathbb{A}_{31}=-\dot{H}_{m}^{(1)}\left(k_{0} R\right) \frac{\Pi\left(k_{0}\right)}{\cosh k_{0} h}, \quad \mathbb{A}_{33}=M_{1}^{\prime} \sum_{q=0}^{n_{q}} \dot{J}_{m}\left(\gamma_{1} \hat{k}_{1_{q}} R\right) \frac{\Gamma_{01_{q}}}{\cosh \hat{k}_{1_{q}} h}, \quad \mathbb{A}_{34}=M_{1}^{\prime} \sum_{q=0}^{n_{q}} \dot{Y}_{m}\left(\gamma_{1} \hat{k}_{1_{q}} R\right) \frac{\Gamma_{01_{q}}}{\cosh \hat{k}_{1_{q}} h}, \\
& \mathbb{A}_{43}=\sum_{q=0}^{n_{q}} J_{m}\left(\gamma_{1} \hat{k}_{1_{q}} R_{2}\right) \frac{\Gamma_{1_{q} 2_{e}}}{\cosh \hat{k}_{1_{q}} h}, \quad \mathbb{A}_{44}=\sum_{q=0}^{n_{q}} Y_{m}\left(\gamma_{1} \hat{k}_{1_{q}} R_{2}\right) \frac{\Gamma_{1_{q} 2_{e}}}{\cosh \hat{k}_{1_{q}} h}, \quad \mathbb{A}_{45}=-J_{m}\left(\gamma_{2} \hat{k}_{2_{e}} R_{2}\right) \frac{\Pi\left(\hat{k}_{2_{e}}\right)}{\cosh \hat{k}_{2_{e}} h}, \\
& \mathbb{A}_{53}=-M_{1}^{\prime} \dot{J}_{m}\left(\gamma_{1} \hat{k}_{1_{q}} R_{2}\right) \frac{\Pi\left(\hat{k}_{1_{q}}\right)}{\cosh \hat{k}_{1_{q}} h}, \quad \mathbb{A}_{54}=-M_{1}^{\prime} \dot{Y}_{m}\left(\gamma_{1} \hat{k}_{1_{q}} R_{2}\right) \frac{\Pi\left(\hat{k}_{1_{q}}\right)}{\cosh \hat{k}_{1_{q}} h}, \quad \mathbb{A}_{55}=M_{2}^{\prime} \sum_{e=0}^{n_{e}} \dot{J}_{m}\left(\gamma_{2} \hat{k}_{2_{e}} R_{2}\right) \frac{\Gamma_{2_{e} 1_{q}}}{\cosh \hat{k}_{2_{e}} h},
\end{aligned}
$$

where $M_{1}^{\prime} \equiv n_{1}+M_{1}$ and $M_{2}^{\prime} \equiv n_{2}+M_{2}$. Other elements (i.e. $\mathbb{A}_{15}, \mathbb{A}_{21}, \mathbb{A}_{25}, \mathbb{A}_{32}, \mathbb{A}_{35}, \mathbb{A}_{41}, \mathbb{A}_{42}, \mathbb{A}_{51}$ and $\mathbb{A}_{52}$ ) are all zeros. The derivatives of the Bessel functions of the first and second kind are defined as

$$
\dot{J}_{m}(a x)=\frac{1}{2} a\left[J_{m-1}(a x)-J_{m+1}(a x)\right] \quad \text { and } \quad \dot{Y}_{m}(a x)=\frac{1}{2} a\left[Y_{m-1}(a x)-Y_{m+1}(a x)\right] .
$$

The derivatives of the modified Bessel function and the Hankel function are respectively defined as

$$
\dot{K}_{m}(a x)=-\frac{1}{2} a\left[K_{m-1}(a x)-K_{m+1}(a x)\right] \quad \text { and } \quad \dot{H}_{m}^{(1)}(a x)=a\left(\frac{m H_{m}^{(1)}(a x)}{a x}-H_{m+1}^{(1)}(a x)\right) .
$$

The functions $\Pi$ and $\Gamma$ have been introduced in (3.19). It also should be noted that the infinite $p, q$ and $e$ need to be truncated to finite numbers, i.e. $n_{p}, n_{q}$ and $n_{e}$. Once the unknown coefficients are solved, the wave solutions for different zones can then be obtained by (4.3), (4.4) and (4.5). 


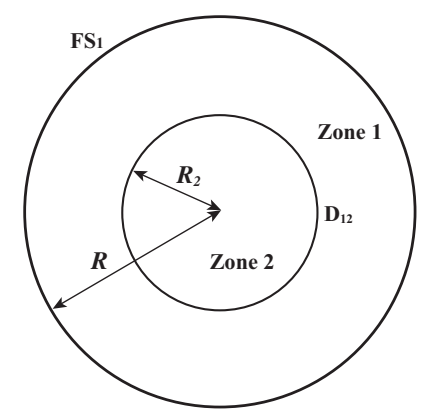

Figure 13: Sketches of a single circular patch with two subzones. $\mathrm{D}_{12}$ shows the interfaces and $\mathrm{FS}_{1}$ indicates the exterior boundary adjacent to the open water region. The cell configuration for Zone 1 is the same as that used in Sec.4.1 $-\ell=9 \mathrm{~cm}, d=3 \mathrm{~cm}$, and the porosities $n=0.9127$. Two special configurations are applied to Zone $2-n=0$ (solid) and $n=1$ (empty).

In the following, the incident waves with period $T=1.00 \mathrm{~s}$ and wave height $H_{\text {inc }}=4.89 \mathrm{~cm}$ under a constant water depth $h=40 \mathrm{~cm}$ are used for demonstration. The radius of Zone 2 is set as $R_{2}=R / 2$. Two special configurations for Zone 2 are applied - (I) an impermeable region $(n=0)$; (II) an empty region $(n=1)$. As shown in Fig.14, the inner zone is assumed as a solid area such that zero fluxes normal to the inner boundary $\left(R_{2}\right)$ is required, i.e. $\partial \phi_{1} / \partial r=0$. For this case, no solutions for Zone 2 is needed. Fig. 15 shows the spatial variation of dimensionless wave amplitude along different profiles. It can be observed that the wave scattering by the solid Zone 2 alters the wave fields in Zone 1 as well as the open water region. For example, along the centerline $Y=0 \mathrm{~m}$, the reflected waves by the solid wall of Zone 2 directly result in an increased wave amplitude as shown in Fig.15a(1). The scattered waves by Zone 2 can also be observed in Fig.15b(4). The wave diffraction converging behind Zone 2 is observed as well. In this case, Zone 1 dissipates both the incident waves and the scattered waves by Zone 2. On the other hand, assuming no cylinders in Zone 2, the dimensionless wave fields are presented in Fig.16. The spatial variation of dimensionless wave amplitude along different profiles is shown in Fig.17 in comparison with the solution for a homogeneous forest (i.e. Liu et al. 2015). Without cylinders in Zone 2, the diffracted waves over the forest region is not as obvious as those for a homogeneous patch. 


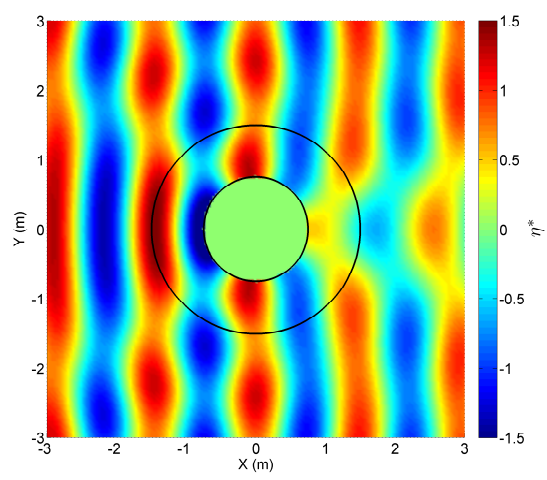

(a) $t=0$ (s)

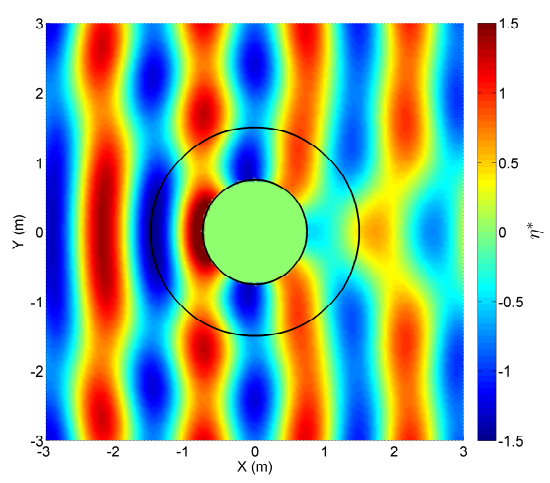

(c) $t=T / 2(\mathrm{~s})$

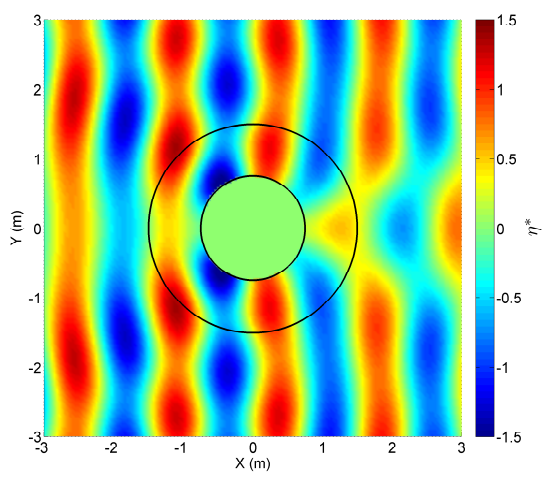

(b) $t=T / 4$ (s)

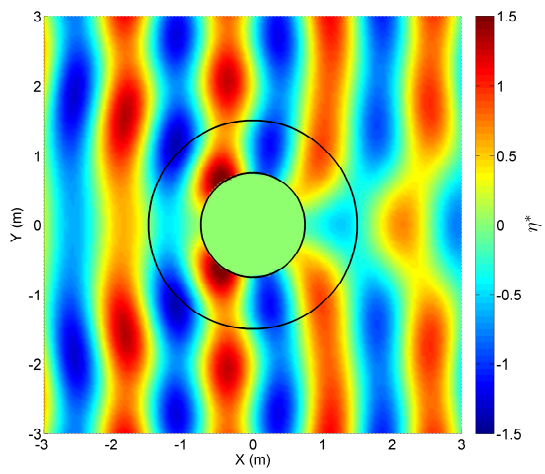

(d) $t=3 T / 4(\mathrm{~s})$

Figure 14: Snapshots of dimensionless free surface elevation over a single circular patch with two subzones within one wave period - Case I. The boundary of the forest patch and the interface between two subzones are indicated by solid lines. The incident wave condition is $T=1.00 \mathrm{~s}$ and $H_{\mathrm{inc}}=4.89 \mathrm{~cm}$ with constant water depth $h=40 \mathrm{~cm}$. 

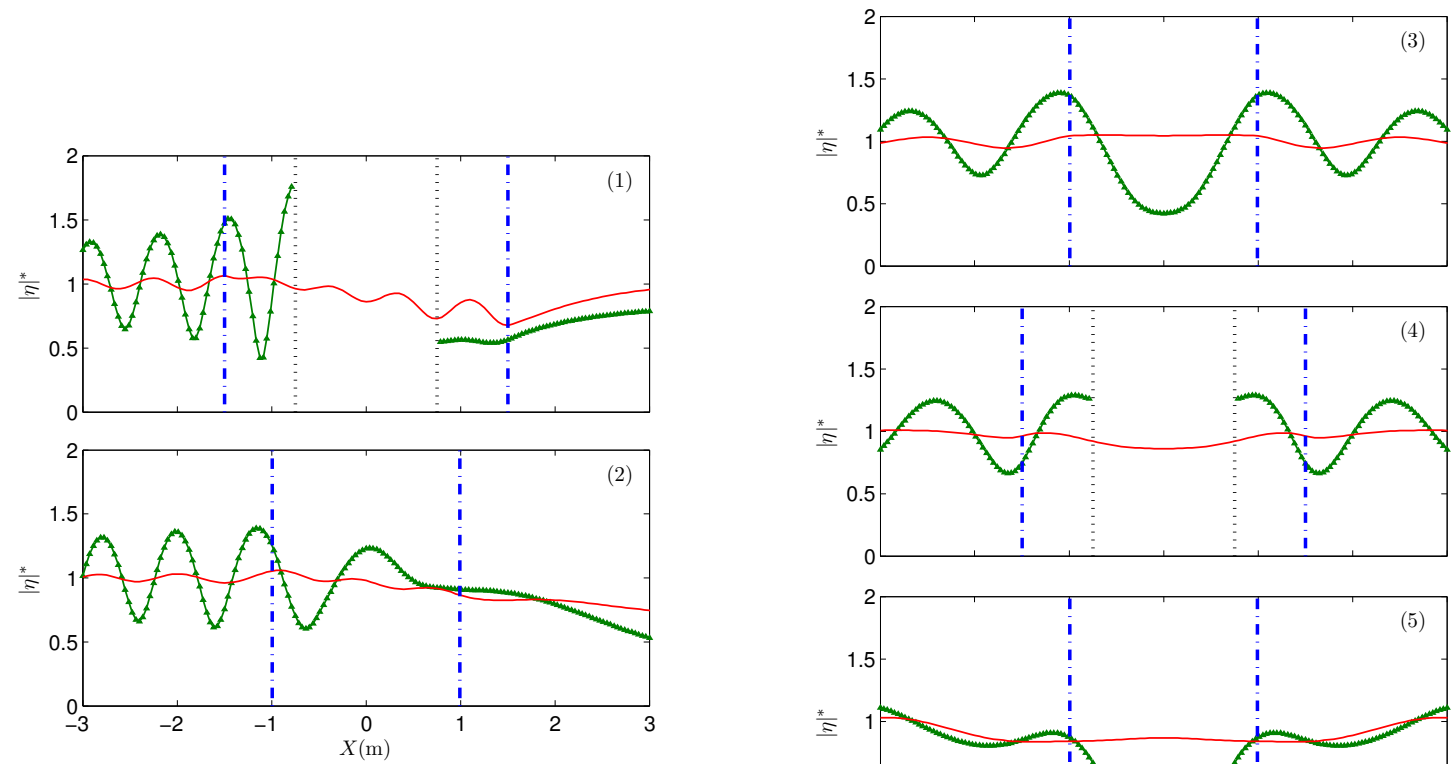

(a)

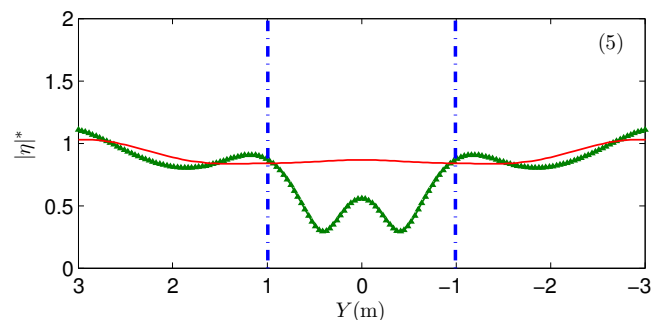

(b)

Figure 15: Spatial variation of dimensionless wave amplitude along different locations - Case I: $(1) Y=0$ $\mathrm{m}$, (2) $Y=1.125 \mathrm{~m}$, (3) $X=-1.125 \mathrm{~m}$, (4) $X=0 \mathrm{~m}$, and (5) $X=1.125 \mathrm{~m}$. The triangle lines show the numerical results; the solid lines indicate the solutions for one homogeneous patch (i.e. Liu et al. 2015). Dot-dashed vertical lines indicate the forest edges and dotted vertical lines show the interfaces between two subzones. The incident wave condition is $T=1.00 \mathrm{~s}$ and $H_{\mathrm{inc}}=4.89 \mathrm{~cm}$ with constant water depth $h=40$ cm. 


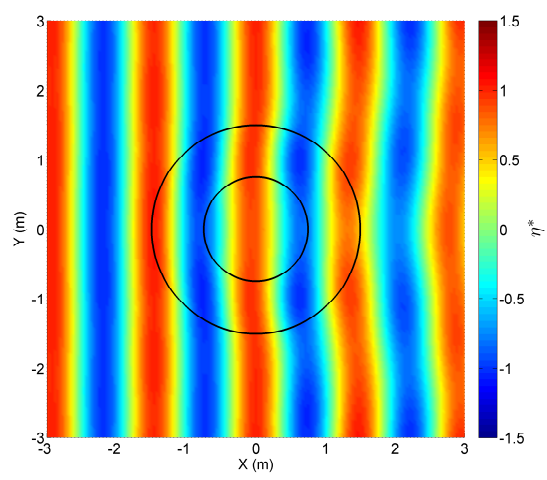

(a) $t=0$ (s)

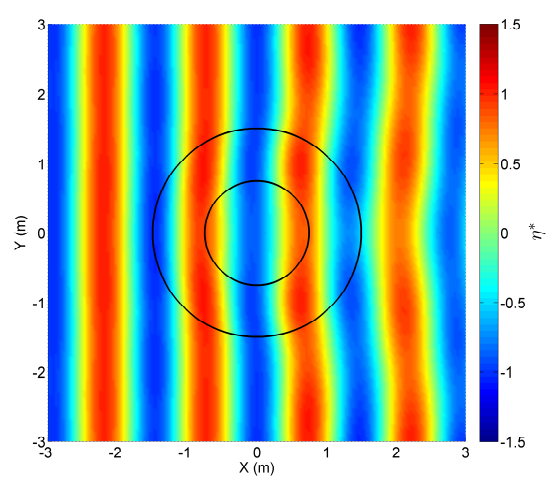

(c) $t=T / 2(\mathrm{~s})$

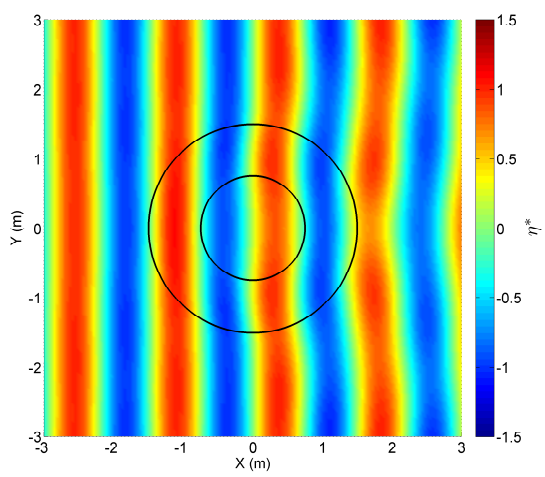

(b) $t=T / 4$ (s)

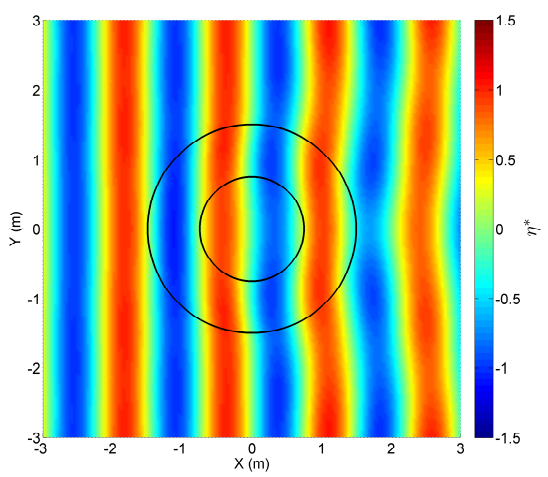

(d) $t=3 T / 4(\mathrm{~s})$

Figure 16: Snapshots of dimensionless free surface elevation over a single circular patch with two subzones within one wave period - Case II. The boundary of the forest patch and the interface between two subzones are indicated by solid lines. The incident wave condition is $T=1.00 \mathrm{~s}$ and $H_{\mathrm{inc}}=4.89 \mathrm{~cm}$ with constant water depth $h=40 \mathrm{~cm}$. 

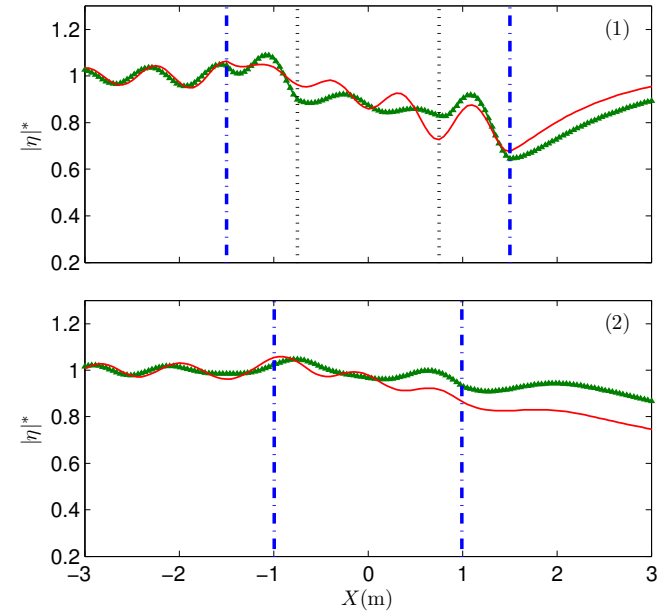

(a)
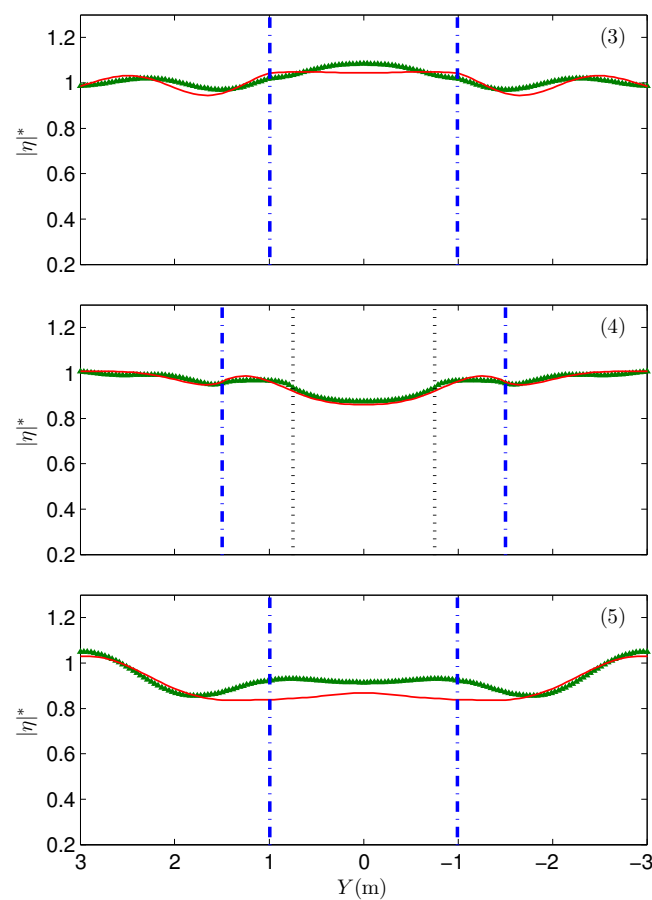

(b)

Figure 17: Spatial variation of dimensionless wave amplitude along different locations - Case II: $(1) Y=0$ $\mathrm{m}$, (2) $Y=1.125 \mathrm{~m}$, (3) $X=-1.125 \mathrm{~m}$, (4) $X=0 \mathrm{~m}$, and (5) $X=1.125 \mathrm{~m}$. The triangle lines show the numerical results; the solid lines indicate the solutions for one homogeneous patch (i.e. Liu et al. 2015). Dot-dashed vertical lines indicate the forest edges and dotted vertical lines show the interfaces between two subzones. The incident wave condition is $T=1.00 \mathrm{~s}$ and $H_{\mathrm{inc}}=4.89 \mathrm{~cm}$ with constant water depth $h=40$ $\mathrm{cm}$.

\section{Concluding remarks}

The theoretical model developed in Liu et al. (2015) has been extended to study the wave propagation through a general coastal forest, which can have multiple patches of arbitrary shape. Each patch can consist of several subzones with different properties (e.g. cell porosity and vegetation types). The multiple-scale perturbation method is applied based on two sharply contrasting length scales (i.e. tree spacing and typical wavelength). The boundary integral equation method is employed to discretize the geometry of forest boundary and to solve the macro-scale problem numerically. In this paper, we consider a subzone as a homogeneous forest region which can be surrounded by other subzones and/or the open water region. To estimate the wave attenuation by the forest region, the eddy viscosity and drag coefficient are both considered as constant bulk quantities within each subzone of the entire forest region and are allowed to be different in different subzones. The energy balance concept and the drag coefficient formula suggested by Liu et al. (2015) are employed to determine the bulk eddy viscosity with the use of the established iterative scheme. A computer program is built up on the basis of the present numerical model, which can be applied to different configurations of forest patches for solving the macro-scale wave solutions with required inputs of configuration as well as its properties.

The single circular forest case studied in Liu et al. (2015) is first used to validate the present numerical model. The present approach is then applied to multiple forest patches as used in Maza (2015)'s experiments. To observe the effects of multiple patches on wave attenuation, we first use the constant value of eddy viscosity for one single circular forest given by Liu et al. (2015) and assign it to all the patches. Iterative scheme is also used to find the eddy viscosity for each patch. The differences among them are within $0.5 \%$. The comparisons between the model predictions and the experimental data are in reasonable agreement. However, 
due to the arrangement of wave gauges and the limited wave conditions in the experiments, the detailed comparison of the variation of wave amplitude within each patch is not possible.

The effectiveness of these two special forest configurations on wave attenuation is compared. As expected, the circular forest has higher efficiency for damping the incoming waves than multiple smaller patches. Furthermore, larger waves yield more wave dissipation for both forest configurations. As a demonstration, we present the numerical results for two different non-circular forest configurations, i.e. one single rectangle and two rectangular patches. We also use a single patch with two subzones to show the numerical results. For all the forest conditions, the wave attenuation and the refraction when propagating through the forest region can both be captured by the present model. More information on the numerical results and the computing model is presented in the supplementary materials.

\section{Acknowledgement}

This research work has been supported by grants from the National Science Foundation (0925711 and 1041541) to Cornell University. Research funding provided by Atkins Center for Sustainable Future and College of Engineering at Cornell University is also acknowledged. CCM was also funded by a Mary Upson Visiting Professorship from Cornell University.

\section{References}

Anderson, E., Bai, Z., Bischof, C., Blackford, S., Demmel, J., Dongarra, J., Du Croz, J., Greenbaum, A., Hammarling, S., McKenney, A., and Sorensen, D. LAPACK Users' Guide. Society for Industrial and Applied Mathematics, third edition, 1999.

Anderson, M. E. and Smith, J. M. Implementation of wave dissipation by vegetation in STWAVE. Technical Report ERDC/CHL CHETN-I-85, US Army Engineer Research and Development Center, Vicksburg, MS, February 2015.

Chakrabarti, A., Chen, Q., Smith, H. D., and Liu, D. Large eddy simulation of unidirectional and wave flows through vegetation. Journal of Engineering Mechanics, 142:04016048, 2016.

Hecht, F. New development in freefem++. Journal of Numerical Mathematics, 20:251-265, 2012.

Horstman, E. M., Dohmen-Janssen, C. M., Narra, P. M. F., van den Berg, N. J. F., Siemerink, M., and Hulscher, S. J. M. H. Wave attenuation in mangroves: A quantitative approach to field observations. Coastal Engineering, 94:47-62, 2014.

Irish, J. L., Weiss, R., Yang, Y., Song, Y. K., Zainali, A., and Marivela-Colmenarejo, R. Laboratory experiments of tsunami run-up and withdrawal in patchy coastal forest on a steep beach. Natural Hazards, 74:1933-1949, 2014.

Isaacson, M. Measurement of regular wave reflection. Journal of Waterway, Port, Coastal and Ocean Engineering, 117:553-569, 1991.

Lara, J. L., Maza, M., Ondiviela, B., Trinogga, J., Losada, I., Bouma, T., and Gordejuela, N. Large-scale 3-d experiments of wave and current interaction with real vegetation. part 1: Guidelines for physical modeling. Coastal Engineering, 107:70-83, 2016.

Lee, J.-J. Wave-induced oscillations in harbours of arbitrary geometry. Journal of Fluid Mechanics, 45: 375-394, 1971.

Lee, J.-J. and Ayer, R. M. Wave propagation over a rectangular trench. Journal of Fluid Mechanics, 110: 335-347, 1981.

Liggett, J. A. and Liu, P. L.-F. The boundary integral equation method for porous media flow. Allen \& Unwin, 1983. 
Liu, P. L.-F., Chang, C.-W., Mei, C. C., Lomonaco, P., Martin, F. L., and Maza, M. Periodic water waves through an aquatic forest. Coastal Engineering, 96:100-117, 2015.

Ma, G., Kirby, J. T., Su, S.-F., Figlus, J., and Shi, F. Numerical study of turbulence and wave damping induced by vegetation canopies. Coastal Engineering, 80:68-78, 2013.

Maza, M. Experimental and numerical modelling of flow interaction with natural ecosystems for coastal protection. PhD thesis, University of Cantabria, 2015.

Maza, M., Lara, J. L., Losada, I., Ondiviela, B., Trinogga, J., and Bouma, T. Large-scale 3-d experiments of wave and current interaction with real vegetation. part 2: Experimental analysis. Coastal Engineering, 106:73-86, 2015.

Maza, M., Lara, J. L., and Losada, I. J. Solitary wave attenuation by vegetation patches. Advances in Water Resources, 98:159-172, 2016.

Mei, C. C. and Vernescu, B. Homogenization methods for multiscale machanics. World Scientific, 2010.

Mei, C. C., Chan, I.-C., Liu, P. L.-F., Huang, Z., and Zhang, W. Long waves through emergent coastal vegetation. Journal of Fluid Mechanics, 687:461-491, 2011.

Mei, C. C., Chan, I.-C., and Liu, P. L.-F. Waves of intermediate length through an array of vertical cylinders. Environmental Fluid Mechanics, 14:235-261, 2014.

Ozeren, Y., Wren, D. G., and Wu, W. Experimental investigation of wave attenuation through model and live vegetation. Journal of Waterway, Port, Coastal and Ocean Engineering, 140:04014019, 2014.

Truong, M. K., Whilden, K. A., Socolofsky, S. A., and Irish, J. L. Experimental study of wave dynamics in coastal wetlands. Environmental Fluid Mechanics, 15:851-880, 2015.

Williams, A. N. Diffraction of long waves by rectangular pit. Journal of Waterway, Port, Coastal and Ocean Engineering, 116:459-469, 1990.

Williams, A. N. and Vazquez, J. Wave interaction with a rectangular pit. Journal of Offshore Mechanics and Arctic Engineering, 113:193-198, 1991.

Yang, Y., Irish, J. L., and Socolofsky, S. A. Numerical investigation of wave-induced flow in mound-channel wetland systems. Coastal Engineering, 102:1-12, 2015.

Zhang, M., Qiao, H., Xu, Y., Qiao, Y., and Yang, K. Numerical study of wave-current-vegetation interaction in coastal waters. Environmental Fluid Mechanics, 16:965-981, 2016. 